ARTICLE

https://doi.org/10.1038/s41467-019-12745-x

\title{
CRISPR-mediated gene silencing reveals involvement of the archaeal S-layer in cell division and virus infection
}

\author{
Isabelle Anna Zink ${ }^{1,4}$, Kevin Pfeifer ${ }^{1,2,4}$, Erika Wimmer ${ }^{1}$, Uwe B. Sleytr ${ }^{3}$, Bernhard Schuster ${ }^{2} \&$ \\ Christa Schleper (iD ${ }^{1 *}$
}

The S-layer is a proteinaceous surface lattice found in the cell envelope of bacteria and archaea. In most archaea, a glycosylated S-layer constitutes the sole cell wall and there is evidence that it contributes to cell shape maintenance and stress resilience. Here we use a gene-knockdown technology based on an endogenous CRISPR type III complex to gradually silence slaB, which encodes the S-layer membrane anchor in the hyperthermophilic archaeon Sulfolobus solfataricus. Silenced cells exhibit a reduced or peeled-off S-layer lattice, cell shape alterations and decreased surface glycosylation. These cells barely propagate but increase in diameter and DNA content, indicating impaired cell division; their phenotypes can be rescued through genetic complementation. Furthermore, S-layer depleted cells are less susceptible to infection with the virus SSV1. Our study highlights the usefulness of the CRISPR type III system for gene silencing in archaea, and supports that an intact S-layer is important for cell division and virus susceptibility.

\footnotetext{
${ }^{1}$ Archaea Biology and Ecogenomics Division, Althanstraße 14, University of Vienna, A-1090 Vienna, Austria. ${ }^{2}$ Institute for Synthetic Bioarchitectures, Muthgasse 11/II, University of Natural Resources and Life Sciences, A-1190 Vienna, Austria. ${ }^{3}$ Institute of Biophysics, Muthgasse 11/II, University of Natural Resources and Life Sciences, A-1190 Vienna, Austria. ${ }^{4}$ These authors contributed equally: Isabelle Anna Zink, Kevin Pfeifer. *email: christa.schleper@univie.ac.at
} 
$\mathrm{M}$ onomolecular arrays of protein or glycoprotein subunits forming surface layers (termed S-layers ${ }^{1}$ ) represent an almost universal feature of archaeal envelopes and have been observed in species of nearly every taxonomical group of walled bacteria ${ }^{2,3}$. In contrast to the complex bacterial peptidoglycan or the pseudomurein found in some methanogenic archaea, the S-layer constitutes a rather simple cell wall, as it is composed of maximally two different protein species, which selfassemble to highly regular arrays of specific lattice patterns (i.e., p1-p6 symmetries), covering the whole cell ${ }^{3}$. In Gram + and Gram - bacteria S-layer units bind to the peptidoglycan containing layer and lipopolysaccharides of the outer membrane, respectively, hence representing an outermost envelope layer of a complex cell wall structure ${ }^{4}$. This is a major difference to most archaea, where the S-layer serves as the sole cell wall component, directly binding the cytoplasmic membrane ${ }^{2}$. Binding is either achieved by a hydrophobic anchoring as in hyperthermophilic Sulfolobus, or via a covalent lipid-linkage, which seems predominant in halophilic archaea ${ }^{5}$. Forming dome-like protrusions, S-layer proteins create a pseudo-periplasmatic space ${ }^{6}$, which was recently shown to stabilize vital cellular processes, such as ion flux ${ }^{7}$. Although some bacteria spontaneously lose their S-layer under laboratory conditions and even overgrow wild-type cells, spontaneous S-layer null-mutants have never been reported for any archaeon ${ }^{8}$, indicating the importance of the structure to these organisms. All investigated archaeal S-layer proteins are heavily glycosylated and therefore many of the functions of this surface protein might be mediated by their glycans ${ }^{9}$. In particular, loss or alteration of the $\mathrm{N}$-glycan structure caused growth retardation under elevated salt conditions and compromised protein secretion of archaeal glycosylation mutant cells in Haloferax volcanii and Sulfolobus acidocaldarius ${ }^{10-13}$. In some of these mutants, an aberrant or perturbed S-layer lattice was observed, which seemed more susceptible to proteolytic degradation, indicating a protective and stabilizing role of the glycosylation ${ }^{10,12}$. However, as surface proteins can share same $\mathrm{N}$-glycan structures, pleiotropic effects of glycosylation breakdown on additional surface proteins cannot be excluded ${ }^{10,11}$. Only few studies reported about phenotypical changes of cells from which the whole S-layer glycoprotein was removed, which might represent the only approach to pin down its functional role in the cell. A primary function ascribed is maintenance of cell shape ${ }^{14-16}$, as dramatic structural changes were observed in rod-shaped archaea ${ }^{17-19}$, as well as the triangularly-shaped Haloarcula japonica ${ }^{20}$ which all became small, spherical protoplasts after S-layer loss in ion-reduced media. Correspondingly, morphological changes were observed in Haloferax volcanii cells defective in the peptidase ArtA, which failed to remove the transmembrane domain of the S-layer glycoprotein, leading to an instable assembly ${ }^{21,22}$. In Sulfolobus islandicus, deletion of the S-layer genes resulted in stripped or partially stripped cells, and alterations in cell morphology, cell division, and sensitivity to hyperosmotic stress ${ }^{23,24}$. In line with this, the S-layer was also suggested to function as osmoprotectant in Halobacterium salinarum, as reflected in higher osmosensitivity of protoplasts ${ }^{25}$.

As a first barrier, the S-layer potentially shields the cell from the environment, as exemplified by standard transformation protocols for halophilic and methanogenic archaea, which require transient removal of the S-layer in order to facilitate DNA uptake $^{26}$. Correspondingly, it represents a first docking site for a number of extracellular molecules such as nutrients, vesicles, cells, and viruses ${ }^{3,5}$. Indeed, only recently, S-layer glycoproteins of hyperthermophilic archaea have been shown to accumulate iron and sulfur from the environment ${ }^{27,28}$. Also, recent studies revealed S-layer glycosylation to be crucial for cell-to-cell interactions during the mating process of the archaeon Halofera $x^{29}$ and comparative genomics suggest a within-niche variation of glycosylation enabling Haloferax to discriminate between such mating partners and also viruses ${ }^{30}$. Similarly, virus- host interaction between Halorubrum sp. PV6 and HRPV-1 was shown to be mediated by recognition of $\mathrm{N}$-formyl-legionaminic, a sugar residue decorating both the virus capsid protein and the host's Slayer protein ${ }^{31,32}$. However, given the possibility that $\mathrm{N}$-glycan 5 $\mathrm{N}$-formyl-legionaminic moiety is shared by additional surface proteins, the essentiality and indispensability of the S-layer for virus binding is not fully resolved. Even though knowledge of the actual role of the S-layer in virus and vesicle interaction is surprisingly sparse, the S-layer counteracts Sulfolobus spindle-shaped virus 1 (SSV1) egress because it seemed disrupted upon virus exit $^{33}$. Taken together, the specific functions of the S-layer in archaea are still very little understood, and investigations of more S-layer depleted mutants are needed to clearly substantiate its role in the cell.

We have recently established a CRISPR-based post-transcriptional silencing technique for targeted gene knockdown in the hyperthermophilic archaeon Sulfolobus solfataricus ${ }^{34,35}$. The technique is based on the RNA-degrading CRISPR type III system, natively encoded in Sulfolobus species, which is reprogrammed to target host mRNA, instead of virus RNA. By transforming a miniCRISPR (miniCR) expressing synthetic small CRISPR RNAs (crRNAs) that matched a host mRNA at one or different positions (i.e., protospacers), we and others have efficiently and gradually silenced genes in different Sulfolobus species to different levels ${ }^{34-37}$.

In this study, we applied the miniCR-based silencing technique to knockdown the S-layer gene slaB (SSOP1_0371), encoding the membrane-anchoring S-layer subunit in S. solfataricus. SlaB proteins are directly held in the membrane by a C-terminal helix domain and form stalk-like protrusions interacting with the outermost S-layer sheath composed of SlaA proteins (cf. Fig. 1a) ${ }^{38}$. The proteins constitute a $\mathrm{p} 3$ symmetry, and interact with other protein components of the cell envelope $e^{6,39-41}$. We show that $75 \%$ silencing of $s l a B$ expression leads to a partial loss and peeling off of S-layer units leading to a severely changed phenotype while higher silencing levels were not tolerated. Our study reveals important functions of the S-layer in S. solfataricus, and highlights the usefulness of the CRISPR-based silencing technology in archaea.

\section{Results}

Construction of miniCRISPR loci (miniCRs) for silencing. For silencing of the slaB gene, three 37-bp target sites (i.e., protospacers: PS1, PS2, PS3) on the slaB mRNA were chosen such that their $3^{\prime}$ flanking sequence (PAS, protospacer adjacent sequence) matched at least $5 \mathrm{nt}$ of the $5^{\prime}$ handle of the targeting crRNA expressed from a miniCR locus, in order to avoid CRISPRmediated DNA degradation (Fig. 1a) ${ }^{34}$. Seven different miniCR constructs were designed matching the three different protospacers in the gene and carrying either single (SB2 and SB3, resp.) or multiple silencing spacers (SB23 and SB123, resp.) (Fig. 1a). In addition, two miniCRs carrying the targeting spacer SB3 two times (SB3 $\times 2)$ and six times (SB3 $\times 6)$, respectively, were designed with the attempt to yield stronger silencing by increasing the dosage of targeting crRNAs (Fig. 1a). Furthermore, a control construct "Ctrl" carrying the miniCR backbone only with two non-binding spacers, was designed. MiniCRs were assembled and inserted into the SSV1 virus-based shuttle vector pDEST-M ${ }^{34}$, generating pDEST-SB vectors. To avoid any unforeseen vectorspecific site effects and to increase the robustness of our results, we decided to use a complementary approach by expressing all miniCRs additionally from the plasmid-based vector pIZ, that we have recently adapted from the pRN1-based plasmid pCMalLacS 
a

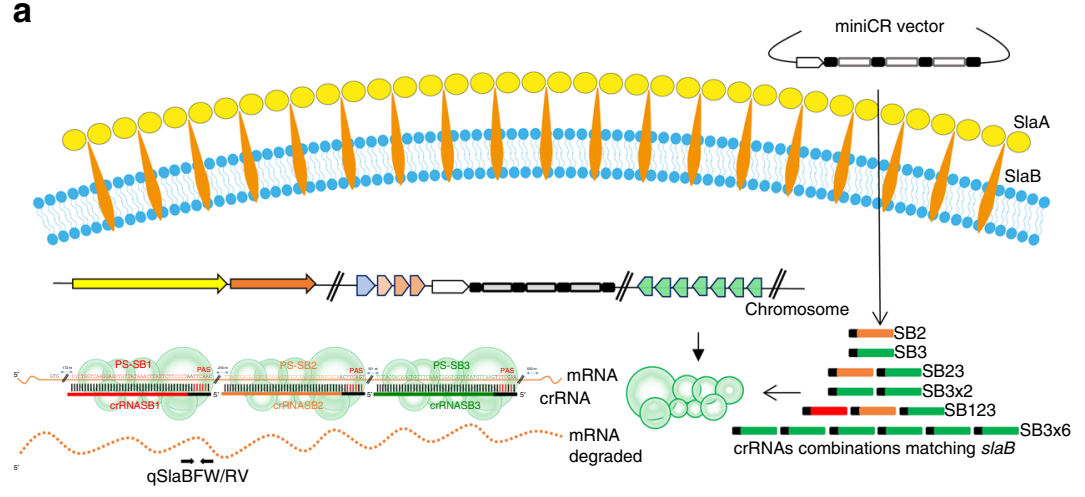

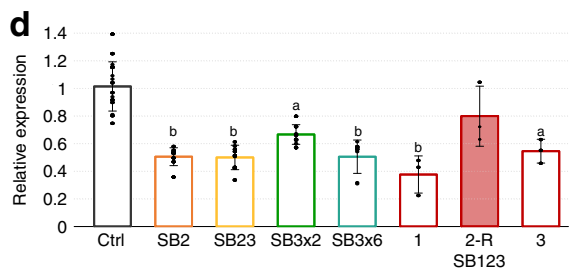

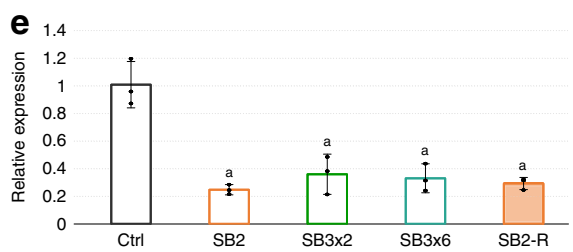

b 1.

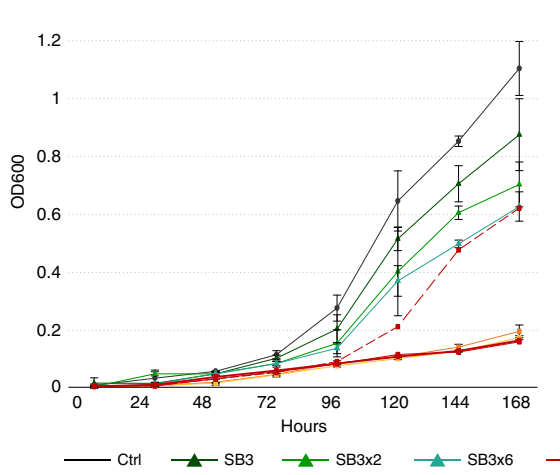

$\mathbf{C}_{1.4}$

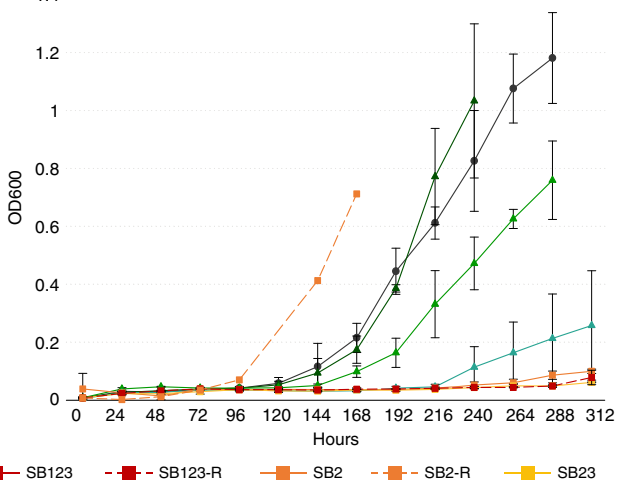

$\mathbf{f}$

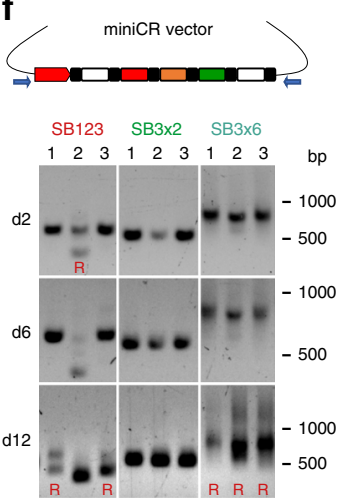

Fig. 1 Silencing of slaB in S. solfataricus. a Schematic representation of the type III silencing pathway used for slaB knockdown. CrRNA assortments expressed from different miniCRISPRs in trans target the slaB mRNA at three possible positions (i.e., protospacer PS1, 2 or 3), each carrying a 3' protospacer adjacent sequence (PAS). Protospacer positions (measured from start codon) and RT-qPCR-primer binding site are indicated ( $\mathrm{qSlaBFW}$ and qSlaBRV). $\mathbf{b}$ Optical density increase of pDEST-SB-silenced cultures and pDEST-Ctrl control cultures (without targeting spacer). SB123-R represents a reverted $(R)$ culture, which has lost the targeting spacer (cf. Fig. 1d and f). Dark gray (circle): control; dark green (triangle): SB3; green (triangle): SB3 $\times 2$; petrol (triangle): SB3×6; red dash (rectangle): SB123 replicates; red dashed (rectangle) SB123-R; orange (rectangle): SB2; yellow (rectangle): SB23. Error bars, mean \pm SD ( $n=3$, i.e., three different plaques picked and inoculated into liquid medium). c Optical density increase of plZ-SB-silenced primary cultures and pIZ-Ctrl control cultures. Dashed orange line (SB2-R) represents a reverted (R) miniCR-SB2 culture, which arose by mutation of the targeting spacer upon culture transfer (cf. Fig. 1e). Color code/line markers and SD are defined as in $\mathbf{b}$. d RT-qPCR on total RNA extracts of pDEST-SB-silenced cultures harvested at $\mathrm{OD}_{600}=0.1$. SlaB mRNA was quantified by qSlaBFW and qSlaBRV primers relative to the reference gene SSOP1_3283

(glyceraldehyde-3-phosphate dehydrogenase); Filled bars = reverted culture (R) (cf. 1c); Lower case letters indicate significant differences to Ctrl (twotailed $t$ test, $n \geq 3, p \leq 0.007$ ). Error bars, mean \pm SD (at least three biol. and tech. replicates). e RT-qPCR on total RNA extracts of plZ-SB-silenced cultures at $\mathrm{OD}_{600}=0.1$. SlaB mRNA was quantified by qSlaBFW and qSlaBRV primers relative to the reference gene SSOP1_3283 (gapN-3); Filled bars $=$ reverted culture (R); Lower case letters indicate significant differences to Ctrl (two-tailed $t$ test, $n=3, p \leq 0.0137$ ); Error bars, mean \pm SD (three biol. replicates). f PCR products amplified by virus vector-backbone primers (blue) of pDEST-SB123, SB3 $\times 2$, and SB3 6 transformants sampled at 2, 6, and 12 days after plaque inoculation, respectively. Three biol. replicates shown $(1,2,3)$. Double bands and smears indicate loss of targeting spacers. Positions of DNA ladder are indicated (bp). Source data are provided ${ }^{70}$

used for transformation of Sulfolobus acidocaldarius ${ }^{42,43}$. Cells transfected with the virus-derived vectors (pDEST-SB2, $-S B 3 \times 6$, -SB23, and -SB123) produced plaques that were picked and grown as single transfectants for further analyses. For pIZplasmid transformations, single colonies harboring an intact miniCR, were only obtained from controls (Ctrl) or from complementation constructs (see below), never from cells transformed with silencing miniCRs only (Supplementary Fig. 1). Therefore, unless otherwise stated, pIZ-plasmid transformants carrying silencing miniCR obtained from primary cultures were used for further phenotypic studies.

Growth defect in silenced cultures and recovery upon spacer loss. Growth retardation of all transformants carrying a silencing miniCR was observed in liquid cultures (Fig. 1b, c). pDEST-SB virus vector transfectants harboring SB2, SB23, or SB123 were most affected, reaching an $\mathrm{OD}_{600}$ of 0.1 only after $\sim 7$ days in culture (Fig. 1b). In pIZ-plasmid transformants, SB123 and SB23 were instable, i.e., plasmids were repeatedly lost after an incubation time of $\sim 7$ days and cultures did not show any growth (Fig. 1c, Supplementary Fig. 2). In all transformants, growth retardation was more pronounced with the multiplex version SB $3 \times 6$ compared to the corresponding single or double spacer constructs SB3 and SB3 $\times 2$, respectively (Fig. 1b, c). All growth effects seemed construct-specific as similar growth patterns were repeatedly found in independently transformed cultures and regardless of the vector backbone being a virus or a plasmid (Fig. 1b, c). Growth recovery was observed in some cultures carrying miniCRs with strong silencing effects (SB123, SB23, SB2, SB3 $\times 6$ ) upon longer incubation or transfer to fresh medium (examples in Fig. 1b, c dotted lines). These cultures had partly lost or mutated the slaB-targeting spacers of the miniCR locus (Fig. 1f). Loss of the targeting spacers in a subset of the 
a
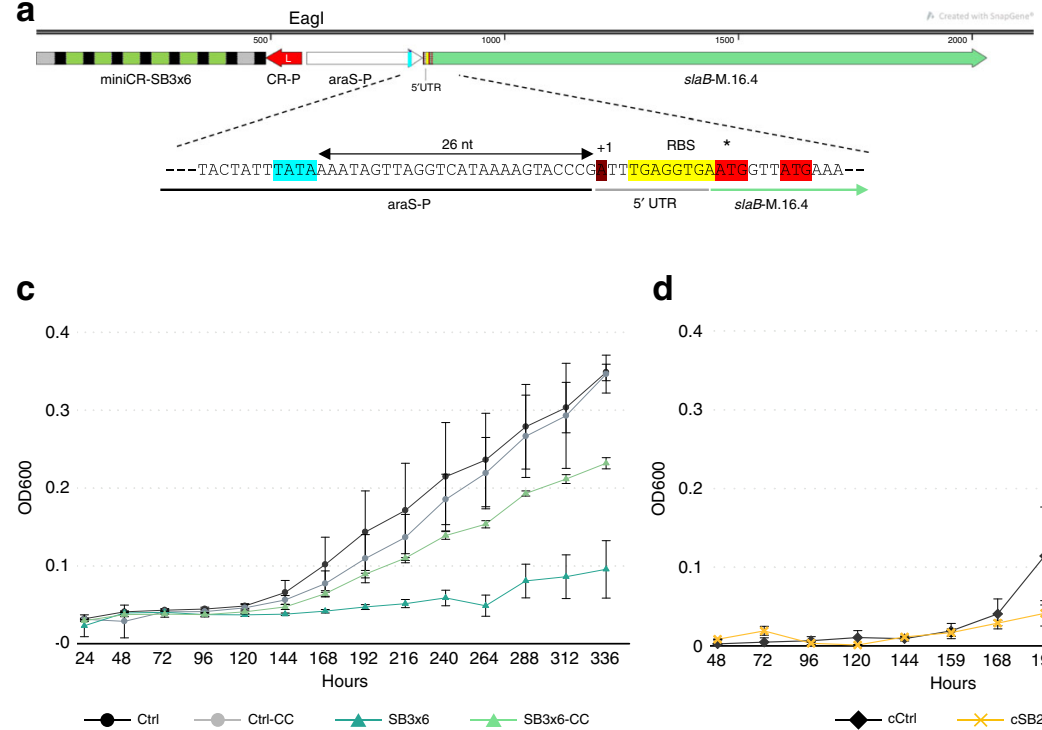

d

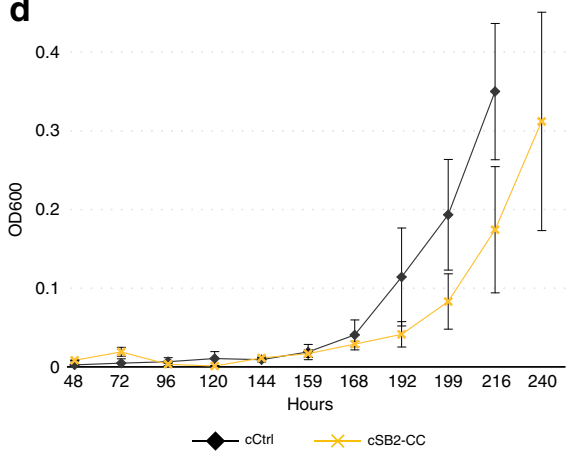

b

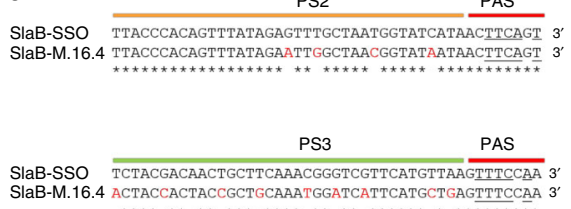

SlaB-SSO TCTACGACAACTGCTTCAAACGGGTCGTTCATGTTAAGTTCCAA
SlaB-M.16.4 ACTACCACTACCGCTGCAAATGGATCATTCATGCTGATTTCCAMA

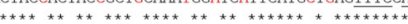

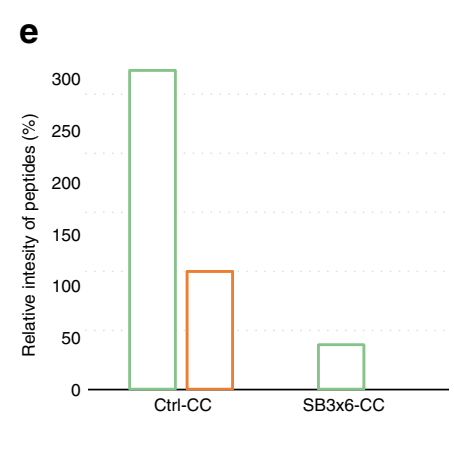

Fig. 2 Complementation of slaB. a Fusion product of SB3×6 miniCR (CRISPR-promoter, red) and the complementation cassette: arabinose-inducible promoter: araS-P (TATA box, blue), 5'-UTR of slaB S. islandicus M.16.4 (M164_1762) including the putative TSS (dark red), a native ribosomal binding site (RBS, yellow) and two potential translation starts (ATG, red). $\mathbf{b}$ Nucleotide sequence alignment of S. solfataricus protospacer 2 and 3 (slaB-SSO) and respective regions on S. islandicus M.16.4 slaB gene (slaB-M.16.4). Asterisks: matching nucleotide; red: mismatches; underlined: PAS- $5^{\prime}$ crRNA handle complementarity. c Optical density increase (arabinose supplemented medium) of plZ-plasmid primary cultures carrying silencing constructs, control constructs (Ctrl) and complementation constructs (-CC), respectively. Dark gray (circle): control; light gray (circle): control-CC; petrol (triangle): SB3 $\times 6$; light petrol (triangle): SB3×6-CC. Error bars, mean \pm SD (three biol. replicates). d Optical density increase (arabinose supplemented medium) of plZ-plasmid cultures recovered from single colonies (prefix " $c$ ") control and complementation constructs. Dark gray (rectangle): control; yellow ( $\mathrm{x}$ ): SB2-CC. Error bars, mean \pm SD (three biol. replicates). e Relative intensity values (MaxQuant) of SlaB peptides of S. solfataricus and S. islandicus detected by mass spectrometry in Ctrl-CC and SB3×6-CC complemented cultures, respectively. Bars in the graph represent the samples in the following order from left to right (in the different cultures): $S$. islandicus $\mathrm{SlaB}$ (light green), S. solfataricus SlaB (brown). Y-axis shows intensity values normalized to Ctrl-CC SlaB-SSO in percent. Source data are provided ${ }^{70}$

population was indicated by multiple bands visible in gel electrophoresis of culture PCR products (Fig. If $\mathrm{d} 2$ and $\mathrm{d} 6$ ), which were identified by sequencing as fully intact and reduced versions of the originally supplied miniCR. Upon further incubation of those mixed cultures, bands corresponding to the intact miniCR disappeared completely (Fig. 1f d12). Spacer loss in cultures with pDEST-viral vectors preferentially seemed to happen precisely at repeat elements, probably through recombination events, leaving the vector including the CRISPR backbone intact. Such cultures are referred to as "revertants" (R), as the expression of slaB reverted to control-like levels (seen by RT-qPCR, Fig. 1d) apparently owing to the destroyed or reduced miniCR. Importantly, SB3 and SB3 $\times 2$ miniCR constructs and control Ctrl were maintained stably in the culture. To analyze whether growth retardation was indeed paralleled by a reduced amount of slaB mRNA, RT-qPCR with specific primers was performed on total RNA extracts of the transformants sampled at an $\mathrm{OD}_{600}=0.1$ (Fig. 1a, Supplementary Table 1) and quantified in relation to the genes SSOP1_3283 (Fig. 1d, e) and the 16S rRNA (Supplementary Fig. 3) used as reference. Figure 1d shows significant silencing of all pDEST-virus vector transfectants in comparison with the Ctrl control cultures. In line with the observed growth retardations, the average silencing effect was $\sim 50 \%$ for SB2, SB23, and intact SB123 cultures and was lower $(\sim 35 \%)$ in SB3 $\times 2$ cultures. As expected, reverted culture SB123-2R, which recovered from growth owing to the loss of the targeting spacers (Fig. 1d, upper panel) did not show any significant reduction of slaB mRNA any more (Fig. 1d, filled bar). In pIZ-plasmid transformants, slaB silencing was assessed only for SB2, SB3 $\times 2$, and SB3 $\times 6$ samples, as the strongest miniCRs SB23 and SB123, were not stably maintained in culture (see above). The silencing trend was similar to the one observable for the pDEST-SB transfectants, however showed an even stronger maximum knockdown level of $\sim 75 \%$ with miniCR-SB2 and SB3×6 (Fig. 1e). Reverted culture SB2-R represented a mixed culture of silenced and escape mutants carrying mutations in the SB2 miniCR spacer, which accumulated after transfer to fresh medium. However, even in the partly reverted cultures, silencing seemed to be still prevalent at the timepoint of sampling as observed in plasmid culture SB2-R (Fig. 1e).

Complementation with $S$. islandicus SlaB recovers phenotype. To analyse whether the silencing effect could be rescued by the expression of a heterologous $\mathrm{SlaB}$ protein in trans, the sla $B$ gene of the close relative S. islandicus (M.16.4_1762) was expressed together with the miniCR from the same vector (Fig. 2a). Among known Sulfolobus species, the SlaB of S. islandicus strain M.16.4 (i.e., SlaB-M.16.4) seemed best suited for complementation, as it showed the highest amino-acid identity ( $88 \%$ via BLAST) to SlaB of $S$. solfataricus (i.e., SlaB-SSO), whereas still containing sufficient divergence on the nucleic acid level (84\% identity via BLAST). Dissimilarity on DNA level was necessary in protospacer regions to avoid targeting of the complementing slaB mRNA by the simultaneously expressed slaB-SSO-specific crRNAs (Fig. 2b). The slaB-M.16.4 gene (including 5'-UTR) was cloned downstream of an arabinose-inducible promoter and inserted into SB2, SB3 $\times 6$, and Ctrl miniCR vectors (virus- and plasmid-based), resulting in Complementation Constructs (-CC) (Fig. 2a). Growth of complemented cultures (SB3×6-CC) has significantly recovered in comparison with the strong growth retardation observable for the non-complemented SB3 $\times 6$ transformants cultivated in parallel (Fig. 2c, Supplementary Fig. 4). Increased cell fitness of the complemented cultures was further reflected by 

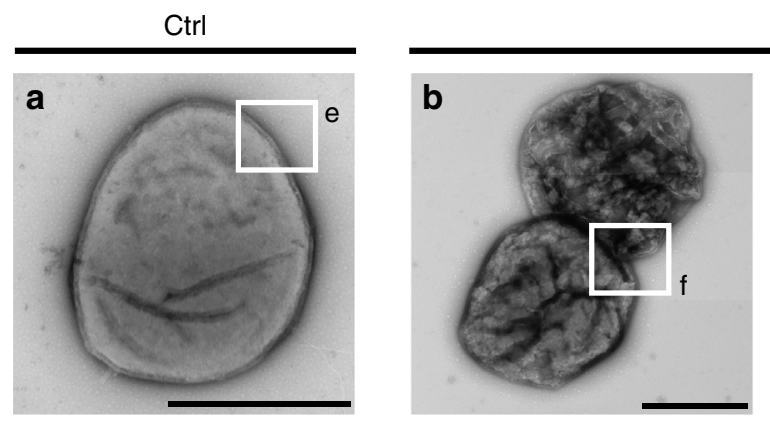

slaB-silenced cultures
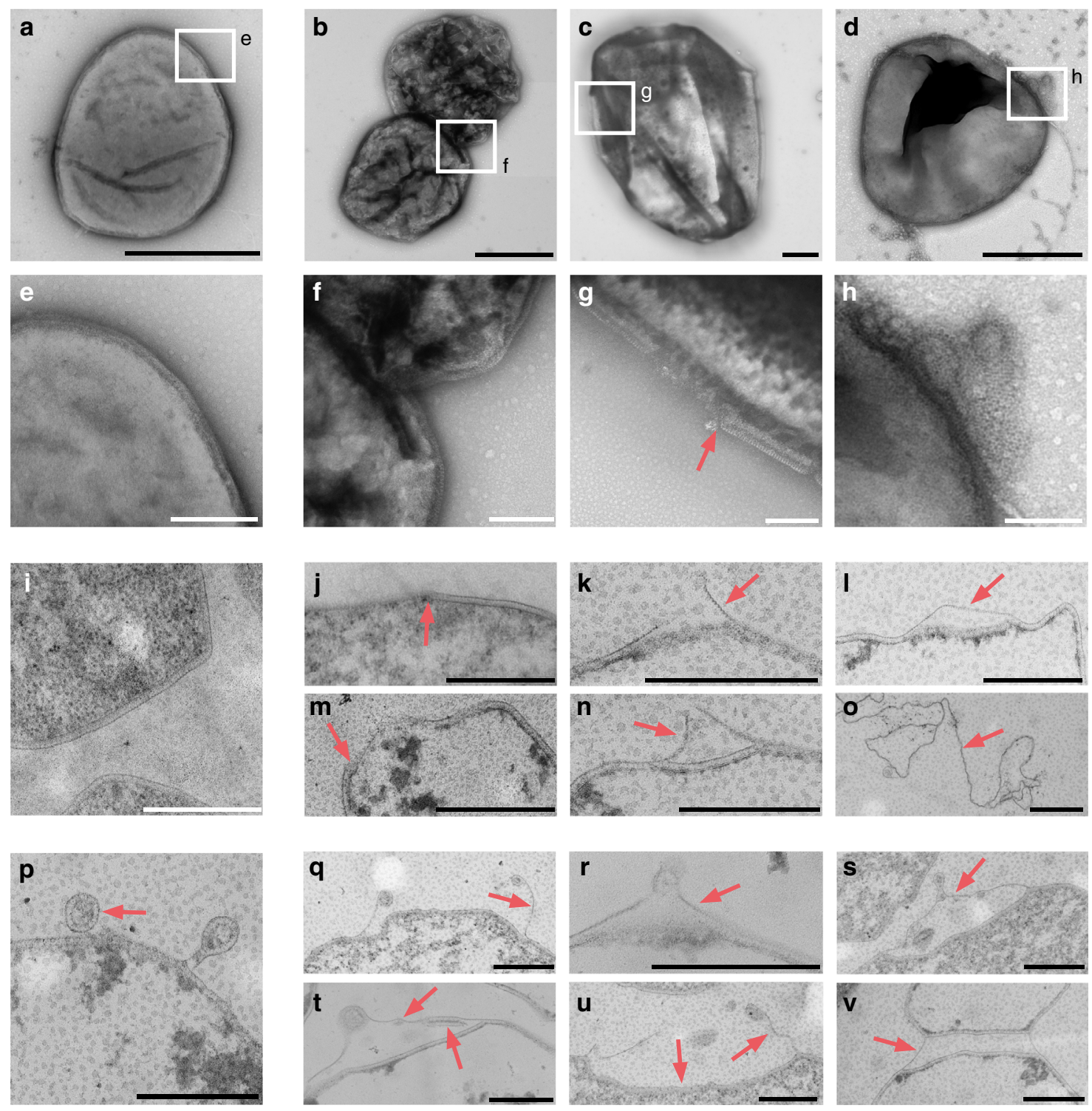

Fig. 3 Electron micrographs of silenced and control (Ctrl) cultures. All cultures (plZ-transformants) were harvested at $O D_{600}=0.1$. a-h Transmission electron micrographs of negatively stained (2\% Uranyl acetate) cells of Ctrl in a and SB3×6 cultures in b-d with indicated areas magnified in e-h. Scale bars: a-d: $1 \mu \mathrm{m}$; e-h: $200 \mathrm{~nm}$. i-o Envelope morphologies of Ctrl in $\mathbf{i}$ and slaB-silenced SB3×6 and SB2 cultures in $\mathbf{j}$-o visualized by transmission electron microscopy of thin sections (contrasted with Uranyl acetate 2\% and Lead citrate 2\%); Scale bar: $500 \mathrm{~nm}$. p-v Vesicle secretion in Ctrl in p and slaBsilenced SB3 $\times 6$ and SB2 cells in $\mathbf{q}-\mathbf{v}$ visualized by transmission electron microscopy of thin sections (contrasted with Uranyl acetate $2 \%$ and Lead citrate 2\%). Red arrows indicate S-layer. Scale bar: $500 \mathrm{~nm}$

the ability of CC plasmid transformants to form single colonies on plates (Supplementary Fig. 1). Moreover, single colonies isolated from SB2-CC transformants showed similar growth rates as Ctrl colonies when transferred to liquid medium (Fig. 2d). To verify expression of SlaB-M.16.4, a band of $\sim 40 \mathrm{kDa}$ of complemented $3 \times 6-\mathrm{CC}$ and Ctrl-CC cultures was recovered from SDS-gels and analyzed by mass spectrometry. SlaB-M.16.4 was found in high quantities ( $10^{6}$ intensity, MaxQuant) in both, Ctrl-CC and SB3 $\times 6$-CC cultures. Data are represented relative to SlaB-SSO intensities of Ctrl-CC samples in Fig. 2e. Notably, SlaBSSO was found in Ctrl-CC cultures only, substantiating silencing of the native slaB in SB3 $\times 6-\mathrm{CC}$ on protein level.

Silencing of slaB affects cell wall integrity and cell shape. Electron micrographs of negatively stained cells revealed heterogeneous populations in the slaB-silenced cultures compared with the homogenous phenotypes found in Ctrl (Fig. 3a). The majority of cells in silenced cultures showed high degrees of surface irregularities (Fig. 3b, f and Supplementary Fig. 5b-d). In addition, a subpopulation of cells was missing fragments of Slayer (Fig. 3c, g) or showed detached S-layer sheets often associated with egressing vesicles (Fig. $3 \mathrm{~d}, \mathrm{~h}$ ). The prevalence of irregular cells in silenced cultures was also reflected in flow cytometry (FC, Supplementary Fig. 6).

Thin sections of Ctrl, SB2, and SB3 $\times 6$ provided further insight into the cell morphology and cell wall structure of silenced cultures. Ctrl cells showed a homogenous phenotype with lobed cells and a prominent cell wall structure as typical for Sulfolobus (Fig. 3i). Contrarily, in silenced cultures, we repeatedly observed cells that were missing segments of S-layer (Fig. 3j, m) or cells 
where the S-layer lattice appeared to be peeling off of cells (Fig. 3k, n) or was partly loosened (Fig. 3l). Such gaps and irregularities in the S-layer structure were detectable in $\sim 8 \%$ of thin-sectioned cells and showed gap widths of varying sizes with an average of $359 \mu \mathrm{m}(S D \pm 271 \mu \mathrm{m}, n=55$, Supplementary Fig. 7). Considering that the observable surface area of thin sectioned cells only represents a tiny fraction of the entire cell surface, the population of affected cells is certainly much higher. Compared with Ctrl (Fig. 3p), similar irregularities in vesicle secretion were observed in silenced cultures, where vesicles appeared to pull off Slayer sheaths from the cell during budding (Fig. 3q-u and Supplementary Fig. 5f). Interestingly, S-layer was observed in surface areas of peeled-off S-layer sheaths (Fig. $3 n$ and $s-v$ ). If Slayer morphogenesis in $S$. solfataricus is comparably fast as in bacteria (500 proteins per second per cell) ${ }^{3}$, these could represent freshly incorporated S-layer proteins. Partially detached S-layer lattice also seemed to connect cells to subcellular structures reminiscent of large "bud-like" appendages (Fig. 3v and Supplementary Fig. 5e, f) recently described for Sulfolobus islandicus ${ }^{44}$. Occasionally, structures resembling S-layer lattice fragments that were completely disconnected from cells were also observed in the preparations (Fig. 3o), similar to those found when S-layer had been chemically stripped from Sulfolobus acidocaldarius ${ }^{45}$.

The various microscopic observations provide evidence that the reduced SlaB level has a pronounced effect on the appearance and shape of the cells and on the cell envelope integrity.

Reduced surface glycosylation in slaB knockdown cultures. To analyze how far the reduction of the SlaB protein influenced the surface glycosylation of the cell, glycosylation profiles of all cultures were analyzed by lectin stains (WGA, Alexa Fluor 488 Conjugate, ThermoFisher Scientific) (Fig. 4, Supplementary Fig. 8, Supplementary Fig. 9). Compared to the Ctrl culture, $60 \%$ and $45 \%$ of cells in the silenced SB2 and SB3 $\times 6$ cultures, respectively, showed decreased fluorescence, indicating a reduction of surface glycosylation (Fig. 4 "low"). The fluorescence profile of the complemented cells SB3 $\times 6-C C$, SB2-CC, and control Ctrl-CC indicated a higher degree of surface glycosylation compared to silenced cultures and Ctrl cells. This shift was most likely caused by the integration of the $S$. islandicus SlaB, which has been predicted to contain more potential glycosylation sites than the $S$. solfataricus protein ${ }^{39,46}$. These observed changes in surface glycosylation suggest $\mathrm{SlaB}$ to be a decisive constituent of the continuous sugar coat of a Sulfolobus cell.

SlaB silencing leads to increased cell size. Phase contrast and electron microscopy revealed dramatic cell size heterogeneity in silenced cultures exhibiting up to fourfold larger cells compared with Ctrl control cultures (Fig. 5a). When measuring cell sizes manually by analyzing phase contrast micrographs of fresh cultures ( $n>300$ for different biol. replicates), we found silenced cells to average at $3 \mu \mathrm{m}$, whereas the regular Ctrl cell size was 1.6 $\mu \mathrm{m}$ (Fig. 5b). Cell sizes of $65 \%$ of counted cells in slaB-silenced cultures were ranging between 2.5 and $8.5 \mu \mathrm{m}$, clearly exceeding the maximum cell size abundantly found in Ctrl cultures ( $>90 \%$ between 1.5 and $2 \mu \mathrm{m}$ ) (Fig. 5b). This trend was reproduced by FC ( $n=10,000$ per biol. replicate), where the FSC-histograms (FSC $=$ forward scatter) were positively skewed in silenced cultures, reflecting the prevalence of larger cells (Fig. $5 \mathrm{c}$ ). To relate abundances of the large cell fractions in the silenced culture to the Ctrl culture, the FSC-histogram of Ctrl cultures was divided into "small cells" (25\% of culture), "average cells" (50\% of culture), and "large cells" ( $25 \%$ of culture). Approximately $40 \%$ of SB $3 \times 6$ and SB2 cells fell into the "large cell" section, whereas complemented cultures showed a significantly lower proportion of larger cells (28\%), similar to Ctrl (Fig. 5c "large"). Enlarged cells occasionally appeared to have a reduced cytosol density in thin sections (Fig. 5a, lower right panel). However, as live-dead staining before thin sectioning showed that both large and regularly-sized cells were indeed viable and contained DNA (Supplementary Fig. 10), we conclude that cell wall depletion destabilized the cells to such a degree that they became more susceptible to damages during the preparation for electron microscopy, as previously observed in other S-layer mutants ${ }^{47}$.

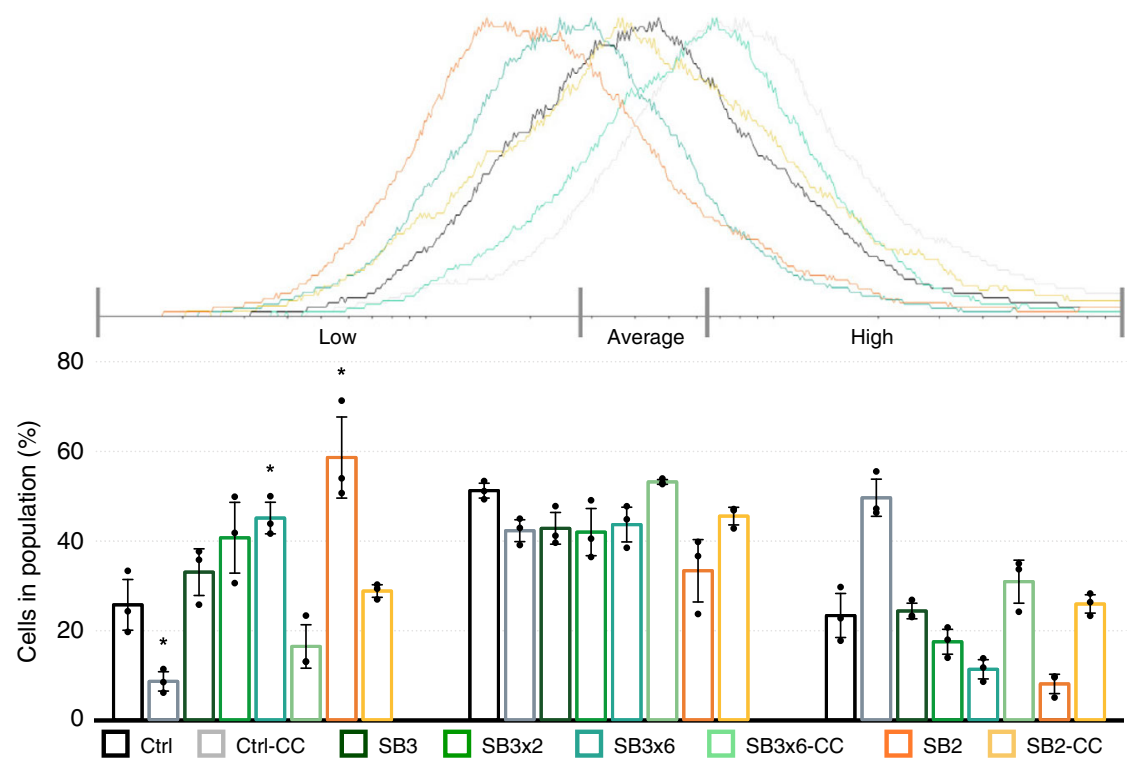

Fig. 4 Surface glycosylation in silenced and control plZ-plasmid cultures. FC-Histogram (fluorescent flow cytometry, created with Flowing Software 2.5.1, 3 biol. repl, $n=10,000$ per replicate) of lectin stained (WGA-Alexa488) cultures harvested at $O_{600}=0.1$. Bar chart represents percent of cells in population showing low, average or high fluorescence (normalized to cell size). Sections were defined according to Ctrl histograms. Bars in the graph represent the samples in the following order from left to right (in every section): Control (dark gray); control-CC (light gray); SB3, (dark green); SB3 2 (green); SB3×6 (petrol); SB3×6-CC (light petrol); SB2 (orange); SB2-CC (yellow). Error bars, mean \pm SD (three biol. repl.). Asterisks indicate significant differences to $\mathrm{Ctrl}$ (two-tailed $t$ test, $n=3, p \leq 0.037$ ). Source data are provided ${ }^{70}$ 
a
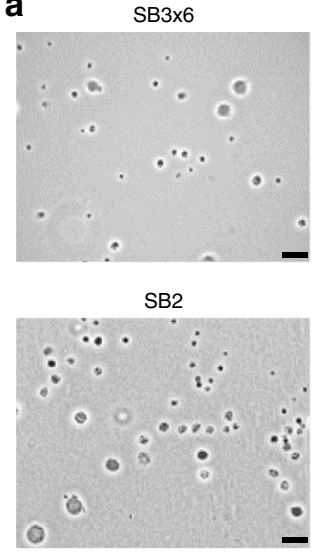

Ctrl

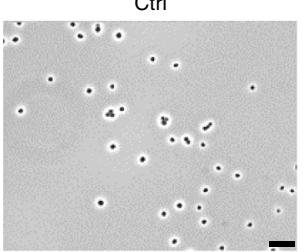

SB3×6-CC

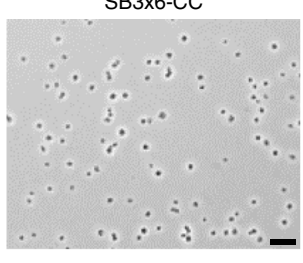

SB2-CC

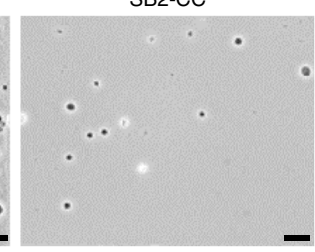

Ctrl-CC

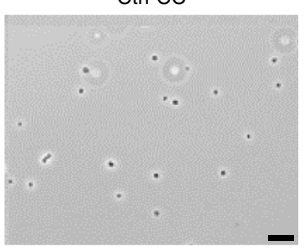

SB3x6
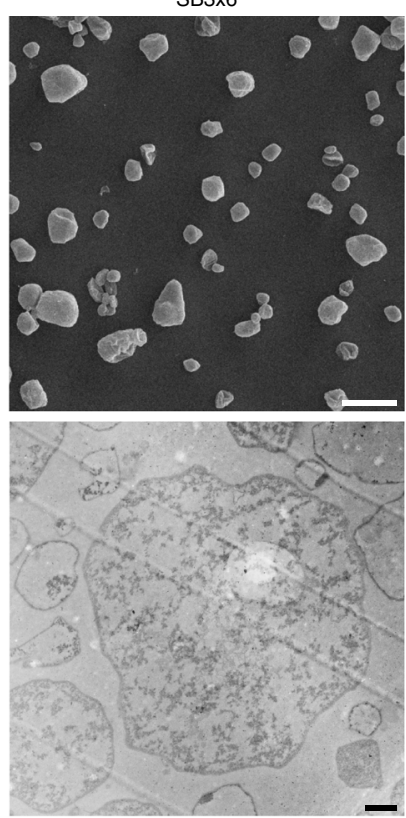

Ctrl
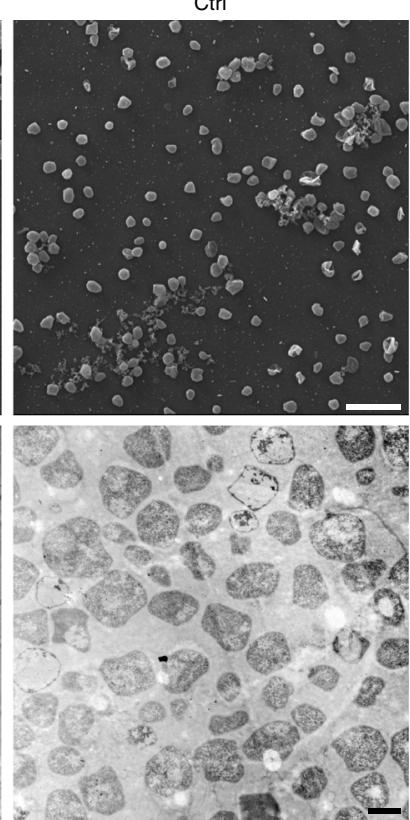

b

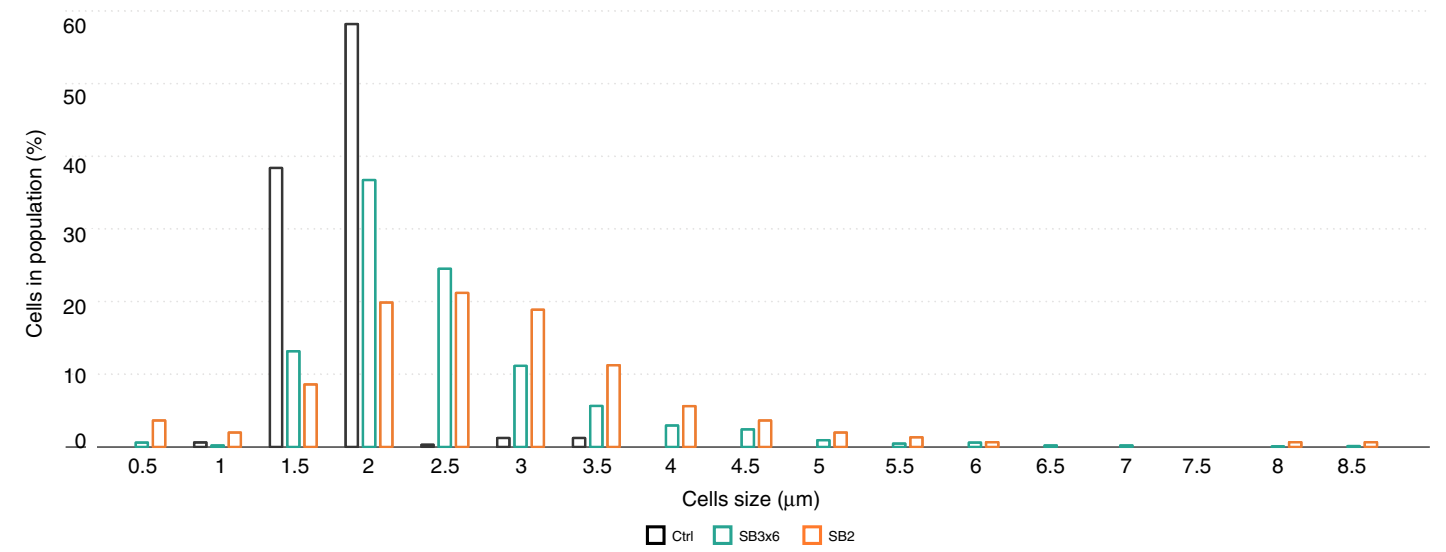

C

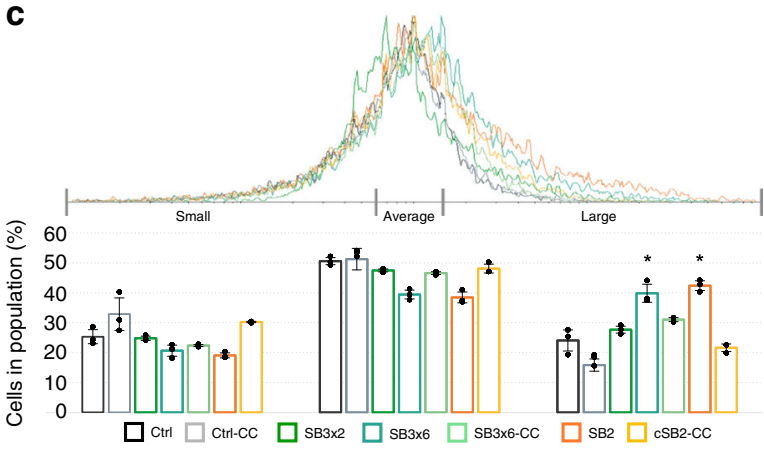

d

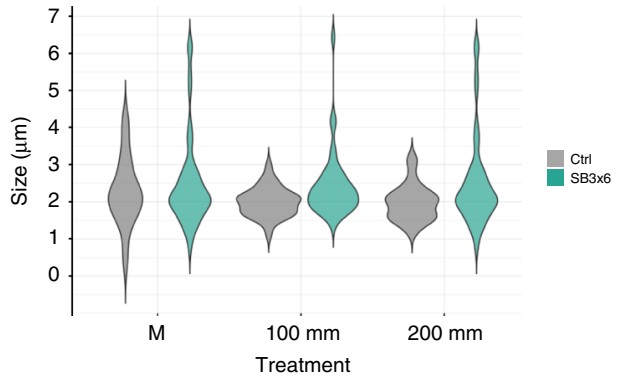

When silenced and control cultures were exposed to increasing $\mathrm{NaCl}$ concentrations, a general decrease of the average cell size was observed, which was slightly higher in SB3×6 (23\% diameter decrease) than in Ctrl cultures (19\% diameter decrease) (Fig. 5d). However, enlarged cells $(7 \mu \mathrm{m})$ persisted over salt-treatments, indicating that they were stable upon hyperosmotic stress (Fig. 5d). Generally, as the observed differences between slaBsilenced and control cultures were relatively small, the S-layer of Sulfolobus might not play a major role in providing osmotic cell stability, at least not upon moderate hyperosmotic changes.
SlaB knockdown affects cell division and chromosome copy numbers. In order to investigate a possible correlation between the large cell phenotype and the measured growth retardation (Fig. 1b, c), fresh cells of silenced and control pIZ-plasmid cultures were harvested precisely at $\mathrm{OD}_{600}=0.1$ and cell numbers as well as average sizes were determined by FC (Fig. 6a). In total, $1.12 \times 10^{7}$ and $1.59 \times 10^{7}$ cells per ml were counted on average in SB2 and SB3 $\times 6$-silenced cultures, respectively, which was around half of the cell numbers measured in Ctrl cultures $\left(3 \times 10^{7}\right.$ cells per $\mathrm{ml}$ ) (Fig. 6a). The lower cell count in knockdown cultures was 
Fig. 5 Cell size distribution in silenced and control plZ-plasmid cultures. a Left: phase contrast micrographs of Ctrl control, SB3×6 and SB2 slaB-silenced (left) and complemented cultures (-CC) (right). Scale bar: $10 \mu \mathrm{m}$. Right upper panel: scanning electron micrographs (gold coated) of slaB-silenced SB3×6 and Ctrl; scale bar: $5 \mu \mathrm{m}$ (magnification $\times 2700$ ). Right lower panel: Transmission Electron micrographs (magnification: $\times 6000)$ of thin sections (contrasted with Uranyl acetate $2 \%$, and Lead citrate $2 \%$ ) of slaB-silenced SB3 $\times 6$ (left) and control Ctrl cultures (right); scale bar: $1 \mu \mathrm{m}$. b Cell size distribution of $\mathrm{Ctrl}, \mathrm{SB} 3 \times 6$, and SB2 cultures $\left(\mathrm{OD}_{600}=0.1\right)$. Bars in the graph represent the samples in the following order from left to right: Control (dark gray); SB3 $\times 6$ (petrol); SB2 (orange). Cell sizes were measured using MicrobeJ software on phase contrast micrographs $(n>300$, different biol. replicates). c FSC-histogram (Forward scatter, flow cytometry, created with Flowing Software 2.5.1) of silenced and control cultures sampled at $\mathrm{OD}_{600}=0.1(n=$ 10,000 per biol. replicate). Bar chart represents percentage of cells in population exhibiting "small", "average" and "large" size (size sections defined according to Ctrl histograms). Bars in the graph represent the samples in the following order from left to right (in every section): Control (dark gray); control-CC (light gray); SB3×2 (green); SB3×6 (petrol); SB3×6-CC (light petrol); SB2 (orange); SB2-CC (yellow). Error bars, mean \pm SD (three biol. replicates). Asterisks indicate significant differences to Ctrl (two-tailed $t$ test, $n=3, p \leq 0.009$ ) $\mathbf{d}$ Salt-stress experiment. Violin plot depicts decrease and increase of abundances of specific cell sizes of untreated (M) plZ-plasmid SB3 $\times 6$ and Ctrl cultures ( $n=50$ cells per replicate and condition), and upon $100 \mathrm{~mm}$ and $200 \mathrm{~mm}$ salt treatment (treatment upon salt addition $=45 \mathrm{~min}, 77^{\circ} \mathrm{C}$, agitating), respectively. Plots represent the samples in the following order from left to right (in each treatment): Control (gray); SB3×6 (petrol). Cell size differences were measured as in b). Source data are provided ${ }^{70}$
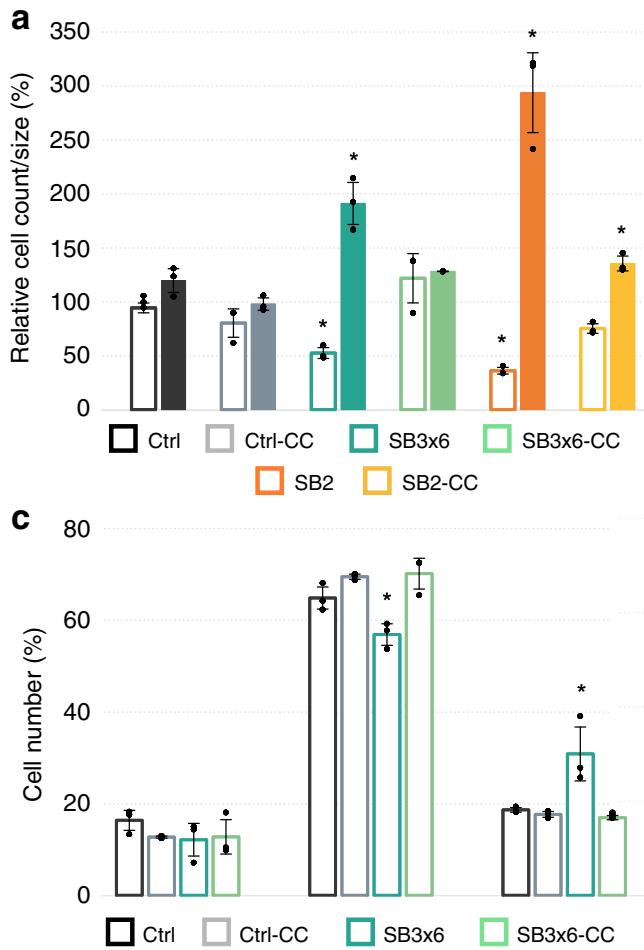
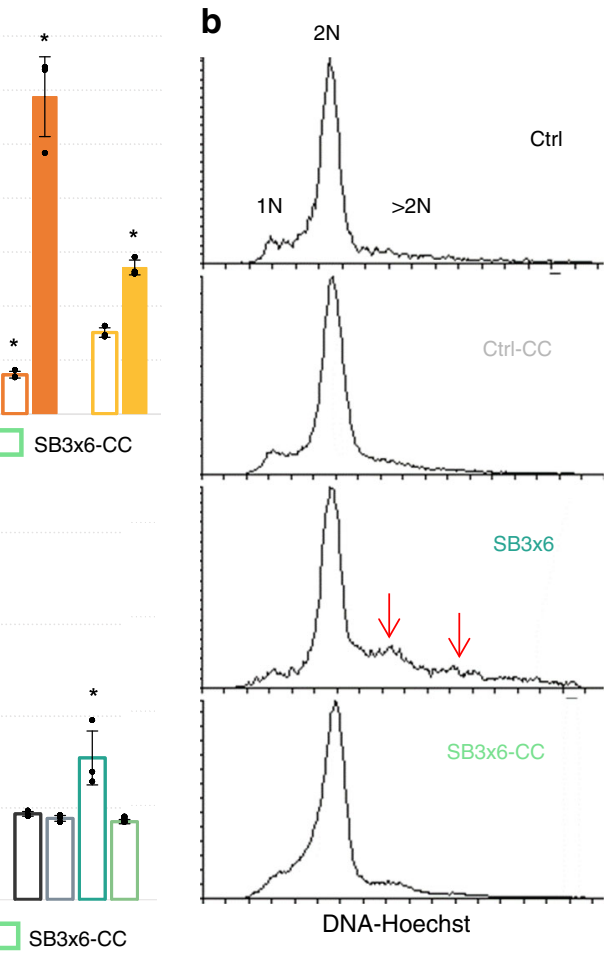

Fig. 6 Correlation of cell count, size, and DNA content in silenced and control cultures of plZ-cultures. a Differences in cell counts (cells per ml) determined by flow cytometry (calculated from flow rate) and cell size (FSC, $n=10,000$ per biol. repl.) of same cultures harvested at precisely $\mathrm{OD}_{600}=0.1$ and normalized to Ctrl control. Cell counts: empty bars, cell size: filled bars. Bars in the graph represent the samples in the following order from left to right (for both conditions): control (dark gray); control-CC (light gray); SB3×6 (petrol); SB3×6-CC (light petrol); SB2 (orange); SB2-CC (yellow). Error bars, mean \pm $\mathrm{SD}$ (three biol. repl.). Asterisks indicate significant differences to Ctrl (two-tailed $t$ test, $n \geq 3, p \leq 0.018$ ). b Fluorescence histograms of Ctrl, Ctrl-CC, $\mathrm{SB} 3 \times 6, \mathrm{SB} 3 \times 6-\mathrm{CC}$ plZ-plasmid cultures harvested at $\mathrm{OD}_{600}=0.1$ and stained with Hoechst. Peaks represent cell abundances containing one chromosome $(1 \mathrm{~N})$, two chromosomes $(2 \mathrm{~N})$, and more than two chromosomes $(>2 \mathrm{~N})$ in respective cultures (created with Flowing Software 2.5.1). c Percentage of cells containing defined chromosome numbers in each culture (as in b): $1 \mathrm{~N}$ (left bars), $2 \mathrm{~N}$ (middle bars), $>2 \mathrm{~N}$ (right bars). Bars in the graph represent the samples in the following order from left to right (in every section): Control (dark gray); control-CC (light gray); SB3×6 (petrol); SB3×6-CC (light petrol). Error bars, mean $\pm S D$ (three biol. repl.). Asterisks indicate significant differences to Ctrl (two-tailed $t$ test, $n \geq 3, p \leq 0.049$ ). Source data are provided 70

paralleled by an increased average cell size (FSC, $n=10,000$ ), which was over one third $(\mathrm{SB} 3 \times 6)$ larger or even over double (SB2) the size of a regular Ctrl cell (Fig. 6a), reflecting size ratios shown above (cf. Fig. 5b). Notably, cell counts, and sizes of complemented cultures were in the range of the Ctrl controls (Fig. 6a). These results indicate that the moderate optical density increase measured in silenced cultures (Fig. 1b, c) reflected growth in cell width rather than cell division. Furthermore, slaB silencing seemed to affect chromosome copy numbers, as slaBsilenced cultures carried a significant proportion of cells that contained more than two chromosomes ( $>2 \mathrm{~N}$ ) (Fig. 6c). This proportion was visible as fluorescent signals in areas designating $3 \mathrm{~N}$ and $4 \mathrm{~N}$ ploidy in FC-Hoechst histograms of SB3 $\times 6$ cultures (Fig. 6b, Supplementary Fig. 11), which differed from described $1 \mathrm{~N}-2 \mathrm{~N}$ patterns of exponentially growing Sulfolobus ${ }^{48}$. Contrarily, histograms of both control and complemented cultures exhibited $1 \mathrm{~N}-2 \mathrm{~N}$ patterns only, without distinct $>2 \mathrm{~N}$ peaks (Fig. 6b). Increased cell size paralleled by increased DNA content in slaB-silenced cells indicated a collapse of cell partitioning and suggested a significant role of an intact S-layer in cell division. 

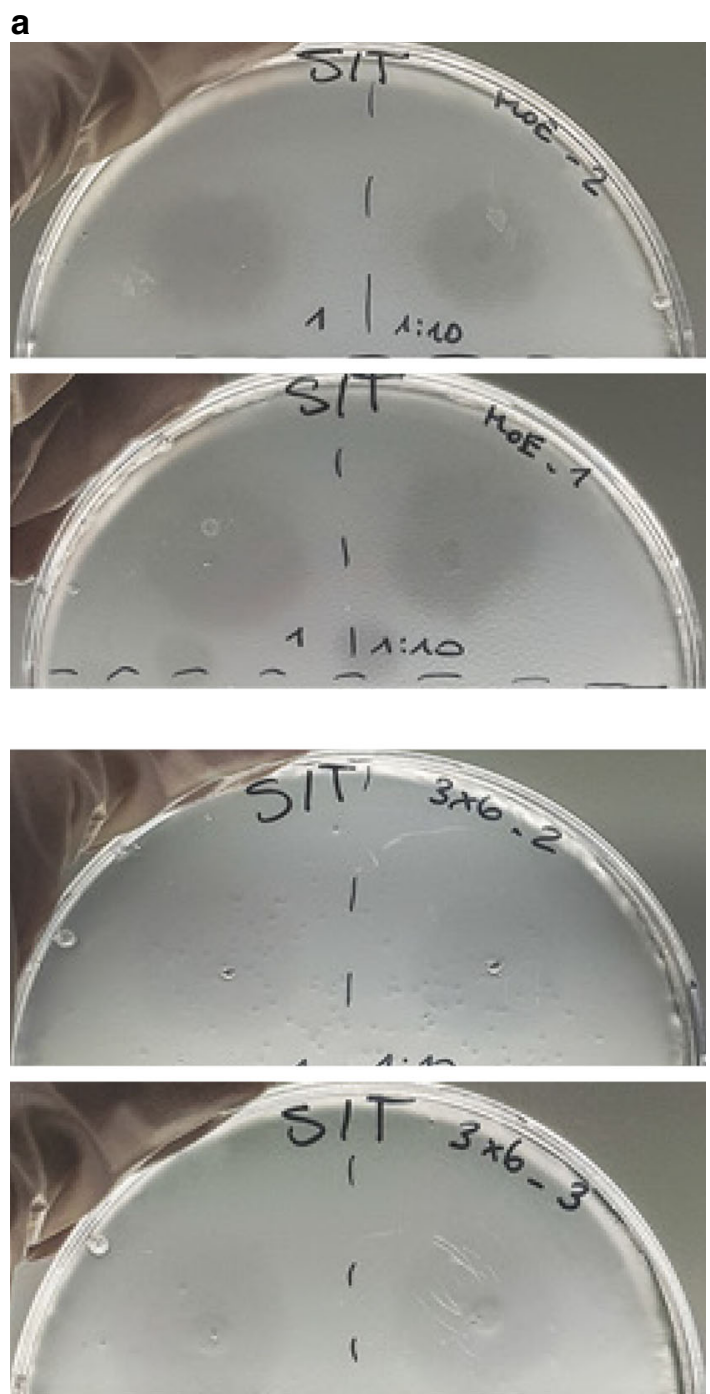

b

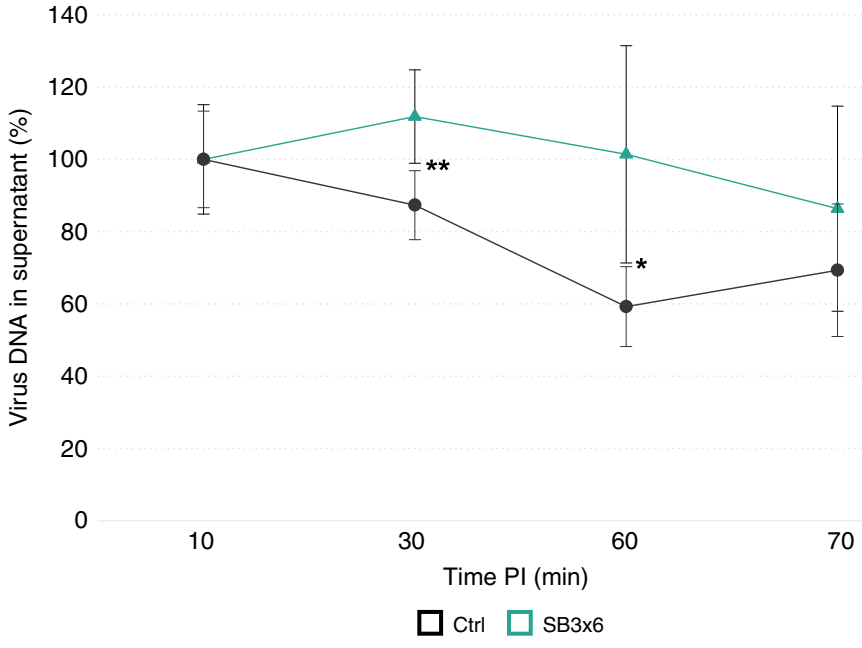

C

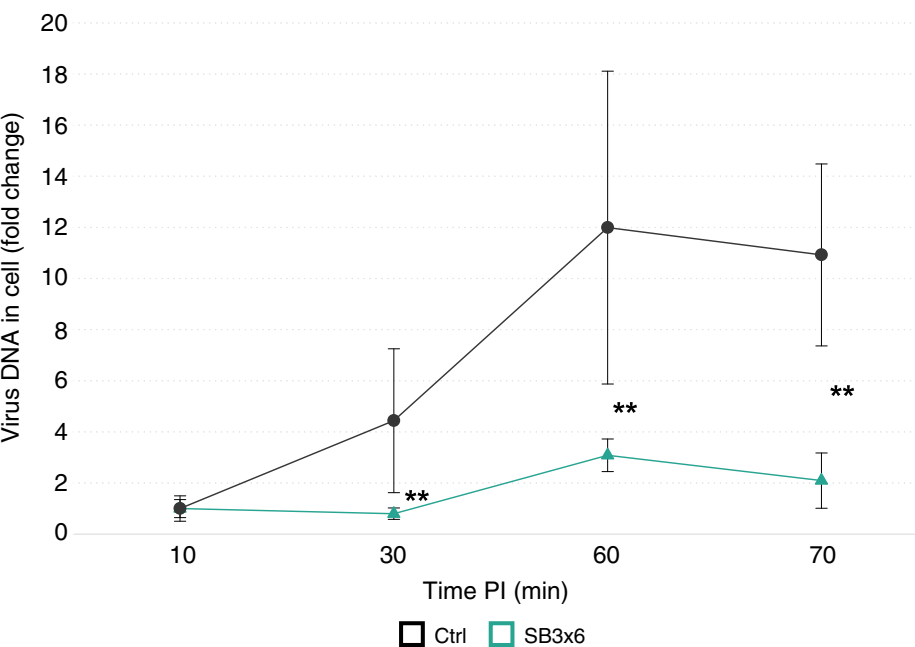

Fig. 7 Infection assays on slaB-silenced and control cultures. a Spotting assay using different dilutions of purified SSV1 virions on cells transformed with plZCtrl (upper panels) and pIZ-SB3×6 (lower panels), respectively. Two biological replicates presented (the same virion sample was used for the different overlays). b Relative amounts of extracellular virus DNA measured at certain timepoints postinfection (PI) in supernatants of plZ-SB3 6 (petrol, triangle) and pIZ-Ctrl cultures (dark gray, circles), which have been challenged with SSV1 virions at MOI 0.1. Virus copies were determined by qPCR using D-291 FW/RV primers (Supplementary Table 1) and normalized to cell count in the individual samples and to the initial virus added. Percentage in relation to timepoint $10 \mathrm{~min} \mathrm{PI}$ is shown. Error bars, mean \pm SD (three biol. and techn. replicates). Asterisks indicate significant differences to Ctrl (two-tailed $t$ test, $n \geq 3, p \leq 0.0105$ ). c Fold change of increase of virus DNA inside cells in same samples as in $\mathbf{b}$. Virus DNA measurement was conducted as in $\mathbf{b}$; error bars are defined as in $\mathbf{b}$. Asterisks indicate significant differences to $\mathrm{Ctrl}$ (two-tailed $t$ test, $n \geq 3, p \leq 0.0083$ ). Source data are provided 70

SlaB silencing impairs infection by SSV1 virus. To investigate whether $s l a B$ knockdown affected virus-host interactions, infection assays with SSV1 virions were performed. First, spotting assays using different dilutions of SSV1 virion solutions on an overlay of concentrated SB $3 \times 6$ and Ctrl pIZ-plasmidtransformants were conducted. Plaques formed after $48 \mathrm{~h}$ of incubation were of the same size but considerably more turbid on SB3 $\times 6$ overlays than on Ctrl, indicating that slaB-silenced cells were less sensitive to virus infection (Fig. 7a). Noteworthy, plaque morphologies of control cultures were comparable to those generated on Sulfolobus wild-type lawns ${ }^{49}$, excluding any influence of the plasmid backbone on plaque formation. To assess the efficiency of virus uptake over time, we mixed SSV1 virions with SB3 $\times 6$ and Ctrl pIZ-plasmid-transformed cells at a multiplicity of infection (MOI) of 0.1 and incubated the suspensions at $77^{\circ} \mathrm{C}$ under agitation. Samples were taken at regular intervals up to 70 min postinfection (PI) and virus DNA in supernatant and cell pellets was quantified by qPCR. Data were normalized to cell count and to initially added virus. Owing to unequal dispersal of virions in the culture, timepoints before $10 \mathrm{~min}$ PI were not considered and virus uptake was thus compared with $10 \mathrm{~min}$ PI (T10). With $87 \%$ of extracellular virus DNA measured, significantly less virus DNA was detected in control cultures at 30 min PI compared with 10 min PI (Fig. 7b). The number of extracellular virus further decreased in Ctrl samples at $60 \mathrm{~min}$ PI, where only $59 \%$ of the initially measured virus DNA could be detected (Fig. 7b). Contrarily, no significant reduction of extracellular virus DNA compared with T10 was observed for neither of the timepoints PI in SB3 $\times 6$ cultures (Fig. 7b). Complementarily, intracellular virus copies increased fourfold at $30 \mathrm{~min}$ PI in Ctrl cultures, whereas almost no increase was detectable for SB3 $\times 6$ (Fig. 7c). Only after $60 \mathrm{~min}$ PI, SB3 $\times 6$ showed an increase in intracellular virus DNA, which, however, was still significantly lower than the 11-fold increase measured for the same timepoint 
Table 1 Relative amounts of SSV1 virus DNA of silenced and control cultures infected at different MOls

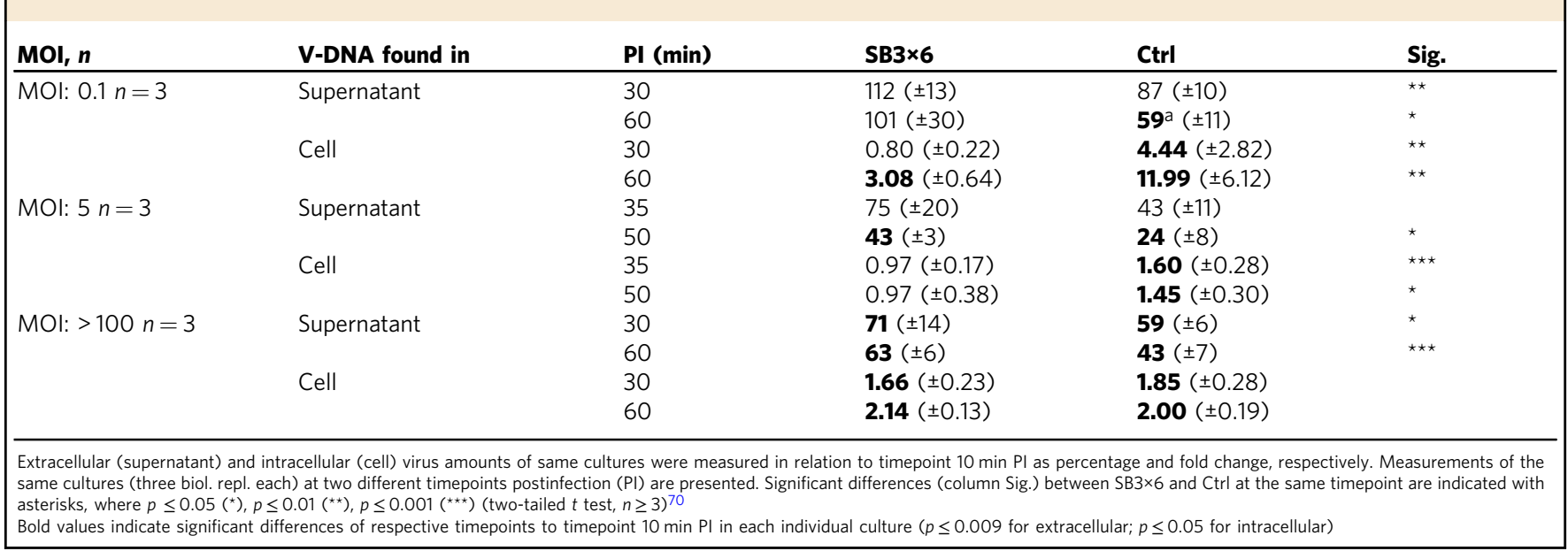

in Ctrl cultures (Fig. 7c, Table 1). At 70 min PI, neither extracellular nor intracellular virus DNA levels changed in SB3 $\times 6$, respectively (Fig. $7 \mathrm{~b}, \mathrm{c}$ ). Contrarily, extracellular virus DNA of control cultures tendentially increased again at $70 \mathrm{PI}$, indicative for a release of freshly produced virions (Fig. 7b). These data show that the efficiency of virus uptake, and therefore, virus susceptibility is significantly higher in Ctrl cultures than in the S-layerdepleted cells at every timepoint examined (Table 1). To investigate if the same effect can be seen at higher virus titers, this experiment was repeated at MOI 5 and MOI > 100 independently, infecting three biological replicates each (Table 1, Supplementary Fig. 12). All infected cultures followed the similar trends as observed for MOI 3, showing a more-efficient decrease of supernatant virus DNA in control cultures, which in all cases, significantly differed from SB3 $\times 6$, at least at $60 \mathrm{~min}$ PI (Table 1). Noteworthy, complementary results were obtained using plaque assays to assess the number of the extracellular virions, as performed for MOI 5, proving the reproducibility of the results using a different experimental approach (Table 1, Supplementary Fig. 12). Apart from MOI > 100, intracellular virus DNA increased over time and was significantly higher in control samples than in SB $3 \times 6$ at all timepoints analyzed (Table 1 ). The high virus titer at MOI $>100$ probably complicates the identification of smaller differences.

\section{Discussion}

In this study, we were able to silence the $s l a B$ gene encoding the membrane-anchoring S-layer protein, up to $75 \%$ using the recently established CRISPR type III-mediated silencing technology 34,35 . Synthetic crRNAs matching the slaB mRNA at different positions were expressed in trans from a miniCR array carried either with a plasmid or a virus vector. Mechanistically, the expressed crRNAs incorporate into a multi-subunit type III complex each and guide it to the complementary protospacers (target regions) on the slaB mRNA, which is subsequently cleaved by the ribonucleolytic action of the $\mathrm{Cmr} 4$ subunits ${ }^{50}$. Apart from disabling DNA degradation ${ }^{51}$, PAS-handle binding as used in our experiments, also inhibits the synthesis of cyclic oligoadenylate by the type III subunit Cas10, which was recently shown to induce unspecific RNA shredding by activation of HEPN family ribonucleases ${ }^{52}$. Therefore, we can preclude any unspecific RNA or DNA degradation when targeting the slaB mRNA using the CRISPR type III-miniCR combo. In eukaryotic RNAi experiments, multiple small RNAs targeting the same gene are used to reject phenotypical changes caused by unspecific off-targeting ${ }^{53}$. Similarly, in our study, two different crRNA (SB2 and SB3) species caused the same phenotypical changes (to same extents when multiplexed) in slaB knockdown cultures, which substantiates the targeting specificity. Furthermore, simultaneous expression of a SlaB from Sulfolobus islandicus M.16.4 in both SB2 and SB3 $\times 6$-silencing experiments rescued silenced phenotypes. This again verified the specificity of the crRNAs and demonstrated that the observed phenotypes indeed resulted from reduced SlaB.

SlaB depletion led to an apparent decrease in binding affinity of the whole S-layer lattice to the cell as seen by electron microscopy showing cells with a discontinuous S-layer coat and with S-layer sheaths peeling off the cell. The protein composition of the detached S-layer sheaths has not been determined in this study. However, SlaA subunits are thought to interact with each other based on their presence as dimers in SDS gels ${ }^{38}$. Moreover, preliminary data of our group provide evidence that SlaA can indeed self-assemble in the absence of SlaB. Thus, the detached sheaths could potentially be formed solely by SlaA proteins, suggesting SlaA subunits to tightly bind to each other but not to the membrane, unless efficiently anchored by the help of SlaB. Detachment of the S-layer sheaths from the slaB-depleted cell envelope was increasingly apparent in cells that showed vesicle protrusions, where the budding vesicle appeared to "pull" the S-layer lattice off the cell wall, a phenomenon which has never been reported for wild-type cells ${ }^{54,55}$. We therefore suggest that a SlaB-depleted Slayer also affects vesicle secretion and coating, as those vesicle poles facing the cell did not seem to be properly covered with the S-layer (cf. Fig. 3r, 3t). The destabilized S-layer lattice had a dramatic impact on cell shape, cell size, and glycosylation. Negative stains revealed cells in slaB-depleted cultures to be more interfolded than control cells, indicating a role in shape maintenance. This complies with earlier observations that lattice folds (i.e., missing S-layer parts) promote the invagination of the membrane, potentially leading to cell shape alterations ${ }^{16}$. Furthermore, the substantial reduction of modifying surface sugar residues in slaB-silenced cultures illustrates that the S-layer majorly contributes to the cell's sugar coat. This coincides with the finding of 31 potential, and five modified $N$-glycosylation sites of SlaA and SlaB proteins, respectively ${ }^{39,56}$. Envelope glycosylation was reported to heavily affect surface properties and motility in mutants of both bacteria and archaea ${ }^{57,58}$ and was speculated to be involved in adaptation to changing environments ${ }^{5}$. Thus, our findings emphasize the importance of a continuous S-layer for the preservation of the physiologically significant sugar envelope.

Silenced cultures revealed an up to fivefold-increase in cell size, as seen by microscopy and flow cytometry. There is another study 
describing size increase of an S-layer stripped archaeon. Methanobacterium thermoautotrophicum protoplasts underwent massive cell shape alterations from rod-shaped to spherical with spheres further increasing in diameter over time. However, the reason for the size increase was not addressed, and Methanobacterium differs from Sulfolobus by the presence of a pseudomurein layer acting as additional cell wall ${ }^{59}$. Notably, cell size increase was often observed in cell division impaired Sulfolobus cells ${ }^{44,60-62}$ where catalytically inactive derivates of cell division (Cdv) proteins were overexpressed, or respective genes were disrupted, respectively. Interestingly, enlarged cells often showed elevated DNA content ${ }^{60,61}$ which was accompanied by a growth defect and cell division failure ${ }^{61}$. This phenotype strongly resembles the slaB-silenced cells in our study, as we observed growth retardation and a significant lower cell count at same optical densities, indicating cellular width-growth instead of cell propagation. Strikingly, we also found a deviant accumulation of DNA copies affecting at least $30 \%$ of the slaB-depleted culture, implying that those cells replicated their DNA, yet failed in undergoing proper fission. Notably, cell sizes, growth, cell count, and DNA content of complemented cultures did not significantly differ from control cells, substantiating that these phenotypes were indeed caused by the reduced S-layer proteins. We therefore conclude that the dramatic size increase accompanied by elevated DNA copies, is caused by impaired cell division due to the depleted S-layer. It has been suggested earlier, that the role of the S-layer in cell division is crucial, potentially by driving membrane invagination through integration of newly synthesized S-layer units at the septum side ${ }^{14-16,20}$. S-layer depletion might destabilize such a process, yet the cell would mount for multiplication, which in turn would lead to accumulation of DNA and other cell components (i.e., lipids). As CdvA was shown to bind to the membrane ${ }^{63}$, a direct interaction with the transmembrane SlaB protein could in theory be possible. Thus, impairment of cell division could also be caused by a disturbed regulation between Slayer and cell division machinery. The fact that S-layer depletion causes phenotypes reminiscent to those of cell division machinery mutants further corroborates an interaction between an intact Slayer and membrane-linked processes.

The attachment site and host cell entry of the widespread Sulfolobus spindle-shaped viruses has remained elusive up to now. Our finding that SlaB-depleted cultures were significantly less susceptible to SSV1 infection than the control cells, indicates an important role of the S-layer on the viral infection cycle. However, it remains to be investigated whether the S-layer functions directly as a virus receptor or secondarily contributes to virus attachment or entry. Contrarily to archaeal filamentous viruses, spindle-shaped viruses were not found attached to cellular appendages, but directly to the cell surface ${ }^{64}$. Also, remarkably high numbers of virions were often observed to attach to the same cell, indicating the cell surface receptor to be highly abundant ${ }^{65,66}$. Thus, as the S-layer accounts for the most abundant surface proteins, we consider it possible, that either SlaA or $\mathrm{SlaB}$ constitute the virus attachment site. Strain-specific glycanmodifications of the S-layer proteins could potentially serve as binding sites, as polysaccharides are common receptors for bacteriophages ${ }^{67}$ and were suggested to play a role in virus susceptibility within the archaeal species Halorubrum sp. PV6 ${ }^{32}$. The S-layer could also secondarily contribute to virus-host interactions, potentially by sustaining the actual surface receptor site. Indeed, the S-layer was found to stabilize other surface proteins, such as the Archaellum and the bindosome-system ${ }^{40,41}$.

In summary, the strong phenotypical effects observed in this study infer that the function of SlaB as S-layer anchor, and the presence of the S-layer as a whole, is important for a proper life cycle of $S$. solfataricus. This is also reflected by the fact that a maximum silencing effect of $75 \%$ could be achieved, whereas stronger silencing constructs were not tolerated. In contrast, we have recently shown that $98 \%$ silencing of the non-essential $\alpha$ amylase gene was possible without any host-induced alterations of the miniCR array ${ }^{35}$. Interestingly, recent studies in the relative S. islandicus M.16.4 showed that slaB gene deletion (or deletion of slaA or a third gene involved in S-layer formation) was possible without causing strong growth delay, even though the majority of the cells showed a partially peeled off (or even fully peeled off) Slayer ${ }^{23,24}$. In those studies, cultivation was performed under nonshaking conditions, and S-layer mutant cells were found clustered in aggregates ${ }^{23}$. Formation of cell clusters and biofilms in Sulfolobus is known to form as a protection mechanism upon stress and to be accompanied by changes in gene expression ${ }^{68,69}$. In our study, cultures were agitated throughout experiments and no cell aggregation was observed. Therefore, it is possible that the strong phenotypes observed in our study might be revealed owing to the harsher cultivation conditions, not promoting cell aggregation, which we believe better resemble the dynamic environment of the natural habitats of $S$. solfataricus, such as bubbling solfataric hot springs.

\section{Methods}

Design of genetic constructs. Three different 37-bp target regions (i.e., protospacers, PS1-3) were selected on the slaB gene (SSOP1_0371) with the following coordinates on the $S$. solfataricus P1 genome (GenBank: LT549890.1): PS1: 338615-338651, PS2: 338870-338906, PS3: 339036-339072. All protospacers were chosen to be flanked by a PAS motif at the $3^{\prime}$-end ${ }^{34}$. MiniCR-spacer sequences SB1-3 were designed to be complementary to the respective protospacer on the sla $B$ mRNA, when transcribed to crRNAs (CRISPR RNAs) (Supplementary Table 1). Spacers for miniCR constructs were assembled by fusion of primer pairs miniCR SB1FW/RV for spacer SB1, miniCR-SB2FW/RV for spacer SB2 and miniCRSB3FW/RV for spacer SB3, respectively, by PCR (Supplementary Table 1) ${ }^{35}$. Furthermore, fusion of primers Ctrl-FW, Ctrl-RV created the non-targeting miniCR array "Ctrl", carrying the miniCR backbone spacers D1 and D5 only ${ }^{34}$ Further assembly of the different spacers to single- and multiplex miniCR constructs were performed as described in ref. ${ }^{35}$ with the difference that a pENTRYTL (truncated leader) vector was used as template in the final PCR step, which carried a truncated version (77 nt-sequence upstream of the first repeat) of the native CRISPR locus D of S. solfataricus P2. Dosage constructs SB3 two and six times, respectively, were constructed by linearizing the pENTRY-SB3 vector using primer pair SlaB3_Lin_FW and SlaB3_Lin_RV within the spacer region. Low-cycle PCR using primer pairs SlaB3_duplex_FW/RV extended the linear fragment by one additional SB3-spacers, generating the SB3 $\times 2$ array ${ }^{35}$. Concatemers of different length formed in PCRs using SlaB3_triplex_FW/RV owing to recombination events between homologous sequence stretches. The SB3 $\times 6$ miniCR was therefore obtained by gel purification of a band at corresponding height (Monarch Gel purification kit, NEB), which was further verified by sequencing. MiniCRs and complementation cassettes (see below) were finally transferred from pENTRY-TL vectors onto the expression vectors pDEST-MJ ${ }^{34}$ and pIZ-GW ${ }^{43}$, respectively, by Gateway in vitro recombination (Invitrogen, Life technologies). pIZ- plasmids carry the pyrEF operon of S. solfataricus P2 for complementation of uracil auxotrophy and were shown to be stably maintained extrachromosomally as a singlecopy plasmid in $S$. solfataricus $\mathrm{P}^{43}$.

For complementation constructs, the slaB gene of S. islandicus M.16.4 (M.16.4_1762, DNA, and S. solfataricus were aligned, which revealed M.16.4 slaB 4 and 10 mismatches in complementary regions of protospacers PS2 and PS3, respectively (cf. Fig. 2b). As mismatches were scattered throughout PS3, and PS2 exhibited two mismatches towards the $3^{\prime}$-end, which is considered important for target cleavage ${ }^{50}$, slaB-M.16.4 was considered divergent enough to avoid crosstargeting of simultaneously expressed SB2 and SB3-crRNAs. For creation of complementation cassettes, first the araS promoter of $S$. solfataricus $P 2^{34}$ was PCR amplified (Phusion Polymerase, ThermoFisher, Scientific) using araFW_EagI and araOH_MSlaB_RV primers (Supplementary Table 1), which added an EagI recognition site to the $5^{\prime}$ and an overhang consisting of the first $25 \mathrm{nt}$ of the slaBM.16.4 sequence (see below) to the $3^{\prime}$-end of the araS sequence, respectively. The sequence chosen for slaB-M.16.4 expression contained the slaB-M.16.4 coding sequence (CDS), as well as the $11 \mathrm{bp} 5^{\prime}$-UTR containing a RBS ${ }^{38}$, as well as the putative terminator sequence downstream to the CDS (genome coordinates: 1615528-1614238, GenBank: CP001402.1). This region was amplified (DNA extracted from S. islandicus M.16.4 obtained from the Rachel Whitaker Laboratory) using primers SlaBM164_FW and SlaBM164_SalI_RV, thereby adding a SalI restriction site to its $3^{\prime}$-end (Supplementary Table 1). AraS and slaB-M.16.4 PCR products were gel-purified (Monarch Gel purification kit, NEB) and subsequently fused at the homologous overlap region using primers araFW_EagI and 
SlaBM164_SalI_RV, in a touch-down PCR reaction (Supplementary Table 1). The thereby assembled complementation cassette was gel-purified, verified by sequencing and further cleaved by EagI-HF (NEB) and SalI-HF (NEB) following manual's instructions. Analogously, respective pENTRY-miniCR-constructs (see above) were cleaved by the same enzymes, respectively, as a EagI and a SalI restriction site are sequentially located $5 \mathrm{bp}$ upstream of the truncated leader promoter (cf. Fig. 2a). Finally, the complementation cassette was ligated upstream to the miniCR cassette into the respective pENTRY vectors (Quick-Ligase, NEB), generating ENTRY-SB2-CC, pENTRY-SB3×6-CC, and pENTRY-Ctrl-CC, respectively which were recovered by E. coli propagation (One Shot TOP 10, ThermoFisher Scientific).

Culturing and transfection. S. solfataricus M18, an uracil-auxotrophic derivate of P1 (DSM 1616, ATCC 35091), was cultivated under shaking conditions at $78^{\circ} \mathrm{C}$, $\mathrm{pH} 3$, in Brock T/S/U medium supplemented with $0.2 \%(+)$ D-Sucrose (S) (Serva) (wt/vol), $0.1 \%$ tryptone (T) (Roth) (wt/vol) and uracil (U) (Sigma Aldrich) at a final concentration of $0.0125 \mathrm{mg}$ per ml $\mathrm{m}^{35}$. For transfection, $50 \mu \mathrm{l}$ of competent cells ${ }^{51}$ were separately mixed with $150 \mathrm{ng}$ of $\mathrm{pDEST}-\mathrm{SB} / \mathrm{Ctrl}$ vector DNA, respectively, and electroporated using 1-mm cuvettes in a Gene Pulser Xcell (Biorad) under the following conditions: $1250 \mathrm{~V}, 1000 \Omega, 25 \mu \mathrm{F}$. After $1 \mathrm{~h}$ recovery, an inverse plaque assay was performed by pouring a $0.4 \%$ gellan gum solution (GELRITE, Roth) containing $2 \mu \mathrm{l}$ of the transfection solution and $10 \times$ concentrated M18 cells on uracil-free Brock-medium plates ${ }^{35}$. After 2 days of incubation, three plaques per transfectant were each isolated, transferred to liquid Brock T/S medium and incubated under the above-described conditions. Transformation of pIZplasmids was performed analogously with modified electroporation-settings: $1240 \mathrm{~V}, 25 \mu \mathrm{F}, 1000 \Omega$. After recovery, the transformation solution was directly transferred to Brock NZ/S liquid medium [0.1\% N-Z-amine AS (NZ) (Fluka Analytics) (wt/vol), $0.2 \%$ D-sucrose (Serva) (wt/vol)] referred to as "primary cultures". Single colony purifications were performed by overlay plating, where $12 \mu \mathrm{l}$ of transformation solutions were mixed with $10 \mathrm{ml}$ of Brock NZ/S medium supplied with $0.4 \%$ gellan gum (GELRITE, Roth) and poured on uracil-free NZ/S plates. Single colonies were isolated after 10 days and were further transferred to Brock NZ/S liquid medium. For incubation of cells transformed with complementation constructs (CC), D-arabinose (BioChemica, AppliChem) instead of sucrose was supplied to growth media to same concentrations.

Growth assays, sampling, and culture PCR. Growth of control and silenced plaque cultures was monitored at OD $(600 \mathrm{~nm})$ (Beckam Coulter, DU $800 \mathrm{Spec}-$ trophotometer), and samples were taken daily to verify the integrity of the miniCRs by culture PCR. For this, $2 \mu \mathrm{l}$ of the culture were used in a PCR reaction (Phusion Polymerase, ThermoFisher, Scientific) together with 406-FW and 406-RV primers binding the vector backbones $\sim 100 \mathrm{bp}$ up- and downstream of the miniCR array (Supplementary Table 1). Entire reaction volumes of each culture PCR sample were loaded on $1 \%$ agarose gels together with an $1 \mathrm{~kb}$ Plus DNA ladder (Thermo Scientific) to determine fragment length. Uncropped gel pictures are provided in the Source Data ${ }^{70}$. In total, $5 \mathrm{ml}$ of $\mathrm{OD}_{600}=0.1$ cultures were harvested cultures for RNA-, DNA-, and S-layer preparations, respectively.

DNA, RNA, and CDNA preparation. DNA and RNA were isolated as described in ref. ${ }^{34}$ followed by further RNAse (Omega, bio-tek) and DNAse (TURBO DNAfree kit, ThermoFisher Scientific) treatment of extracted DNA and RNA, respectively, following the manufacturer's protocol. The integrity of extracted RNA was verified by gel electrophoresis and DNA digestion was checked by PCR. One microgram of RNA was reverse transcribed using ProtoScript II Reverse transcriptase (NEB) according to the manual and further purified (Monarch, PCR \& DNA Cleanup Kit, NEB). Nucleic acid concentrations were determined fluorometrically by Qubit using RNA BR-, DNA BR-, and ssDNA Assay Kit for cDNA, respectively (ThermoFisher Scientific).

Quantitative RT-PCR analysis. All quantitative PCRs were performed using GoTaq qPCR Master Mix (Promega) in an Eppendorf Mastercycler epgradient S relplex2 (Eppendorf). Three technical replicates of each biological replicate of every experiment were measured, respectively. Transcripts of slaB and the SSOP1_3283 housekeeping gene (glyceraldehyde-3-phosphate dehydrogenase), as well as $16 \mathrm{~S}$ rRNA were quantified from same cDNA preparations using primer pairs qSlaBFW/ $\mathrm{RV}, \mathrm{q} 3194 \mathrm{FW} / \mathrm{RV}$, and q16SFW/RV, respectively (Supplementary Table 1). Relative expression of the slaB gene was assessed by calculating the $\Delta \mathrm{C}_{\mathrm{t}}$ of the amplicons of the two primer pairs and normalizing the values to Ctrl control cultures $^{34}$. For infection assays (see below), virion numbers were quantified directly on $5 \mu \mathrm{l}$ of the solution using primers qD291FW/RV (binding ORF A291 of SSV1, see Supplementary Table 1). Standards used in qPCRs were linearized pDEST-MJ vectors (XhoI, ThermoFisher, Scientific) containing the appropriate gene. qPCR efficiency was between 100 and $84 \%$ in all runs.

Light microscopy. For light and fluorescence microscopy, $5 \mu \mathrm{l}$ of fresh cultures were transferred to a microscope slides (Marienfeld superior) and immediately imaged using a Jenoptik microscope camera (ProgRes MF Cool CCD 1.4 M.P) attached to a Nikon ECLIPSE Ni-E. For viability staining, $1 \mathrm{ml}$ of freshly harvested cells were incubated with Syto9 and PI (Invitrogen LIVE/DEAD BacLight Bacterial Viability Kit) according to the manufacturers protocol and imaged (PI: Nikon TRITC Bandpass filter cube; Syto9: Nikon FITC Bandpass filter cube). For Hoechst (Invitrogen H1399) staining, cells were fixed at $4{ }^{\circ} \mathrm{C}$ overnight in $70 \%$ ice cold ethanol, subsequently washed and resuspended in buffer A $(20 \mathrm{~mm}$ TRIS-Acetate buffer $\mathrm{pH}$ 5.5), incubated according to the manufacturers protocol and imaged.

Electron microscopy. Electron micrographs of negatively stained cells were produced from freshly harvested cells $\left(\mathrm{OD}_{600}=0.1\right)$, which were washed once with buffer A and resuspended in $100 \mu \mathrm{l}$ of buffer A. In total, $30 \mu \mathrm{l}$ of the cell suspension were absorbed onto carbon-coated copper grids and negatively stained with $2 \%$ uranyl acetate. For thin sections, $2.5 \%$ glutaraldehyde was added to $2 \mathrm{ml}$ of cells harvested at OD 0.1 and incubated for $2 \mathrm{~h}$. After which the cells were further fixed with $0.1 \%$ osmium tetroxide in $0.02 \mathrm{~mm}$ sodium cacodylate overnight. The pellet of fixed cells was embedded into Low Viscosity Resin (Agar scientific; Agar Low Viscosity Resin Kit) and then cut into $60 \mathrm{~nm}$ thin section (LEICA EM UC6). The sections were absorbed onto formvar coated copper grids and contrasted $(2.5 \%$ Gadolinium acetate, $30 \mathrm{~min}$; $3 \%$ Lead citrate, $8 \mathrm{~min}$ ). Both negatively stained samples and thin sections were analyzed using a Libra 120 (Zeiss). For scanning electron microscopy, late exponential cells were fixed ( $2.5 \%$ glutaraldehyde), washed $(0.02 \mathrm{~mm}$ sodium cacodylate), spotted onto $0.01 \%$-poly-L-lysine-coated glass slides and dehydrated using a graduated ethanol series followed by critical point drying (Leica EM CPD300). The slides were subsequently placed on conductive stubs, gold coated for 60 s (JEOL JFC-2300HR) and analyzed (JEOL JSMIT300).

Flow cytometry. Cell size (FSC), cell granularity (SSC), and glycoprotein (FITC) analysis was performed on a BD FACSCantoII. For FSC and SSC measurements, $50 \mu \mathrm{l}$ of a growing culture were diluted 1:10 in buffer A. For glycoprotein quantification, $20 \mu \mathrm{l}$ of freshly harvested cells were diluted 1:20 in buffer A and subsequently incubated with $40 \mu \mathrm{g}$ per ml Wheat Germ Agglutinin, Alexa Fluor 488 Conjugate (W11261, ThermoFisher Scientific) for $30 \mathrm{~min}$ in the dark at room temperature and analyzed. To measure the DNA content, cells were fixed at $4{ }^{\circ} \mathrm{C}$ overnight in $70 \%$ ice-cold ethanol, subsequently washed and resuspended in buffer A and stained with Hoechst 33342 (Invitrogen H1399) according to the manufacturers protocol. In all, $50 \mu \mathrm{l}$ of the stained cells were diluted 1:10 in the washing buffer and analyzed (MoFlo Astrios. Beckman Coulter).

For cell counting, $20 \mu \mathrm{l}$ of freshly harvested cells were diluted 1:20 in buffer A and cells per $\mathrm{ml}$ were calculated from flow rate $(10 \mu \mathrm{l}$ per min), events recorded per second and recording time (see Source Data file ${ }^{70}$ ). This method was verified by comparison with cell counts by light microscopy.

$\mathrm{NaCl}$ assay. Aliquots of growing $\mathrm{SB} 3 \times 6$ and Ctrl plasmid transformants were adjusted to same cell concentrations (cell number assessed by microscopy) and incubated under normal growth conditions for $2 \mathrm{~h}\left(78^{\circ} \mathrm{C}\right.$, shaking). $\mathrm{NaCl}$ (Roth) was added to the shaking cultures to a final conc. of $100 \mathrm{~mm}$ followed by $45 \mathrm{~min}$ incubation. Consecutively, concentration was increased to $200 \mathrm{mM}$ followed by $45 \mathrm{~min}$ incubation. $5 \mu \mathrm{l}$ of cultures were sampled in triplicates right before $\mathrm{NaCl}$ addition and after each $\mathrm{NaCl}$ incubation period respectively, without interrupting the incubation process. Samples were applied on microscope slides and immediately imaged by phase contrast microscopy (see above). Diameter of cells $(n=50$ per condition and culture) were measured at spots of widest distance on micrographs taken of each culture using Microbej ${ }^{71}$.

S-layer composition analysis. S-layer was purified as previously described ${ }^{38}$ with small alterations, in short: $30 \mathrm{ml}$ of a growing culture at $\mathrm{OD}_{600}=0.1$ were harvested by centrifugations at $2500 \times g$ and $4^{\circ} \mathrm{C}$ for $15 \mathrm{~min}$. The subsequent pellet was resuspended in $2 \mathrm{ml}$ of buffer $1\left(10 \mathrm{~mm} \mathrm{NaCl}, 1 \mathrm{~mm}\right.$ PMSF, $20 \mathrm{~mm} \mathrm{MgSO}_{4}$, $0.5 \%$ sodium lauroylsarcosin, $\mathrm{pH}$ 6) and $10 \mu \mathrm{l}$ DNAse (TURBO DNA-free kit, ThermoFisher Scientific) was added. Subsequently the solution was incubated at $45^{\circ} \mathrm{C}$ for $30 \mathrm{~min}$. Following centrifugation at $13,000 \times g$ for $15 \mathrm{~min}$, the supernatant was discarded, and the procedure repeated. After the second round of centrifugation, the pellet appeared to have two distinct fractions. The top white fraction was carefully resuspended in $2 \mathrm{ml}$ of buffer $2\left(10 \mathrm{mM} \mathrm{NaCl}, 20 \mathrm{mM} \mathrm{MgSO}_{4}\right.$ $0.5 \%$ [wt/vol] SDS, $\mathrm{pH} \quad 8$ ), incubated at $45^{\circ} \mathrm{C}$ for $30 \mathrm{~min}$ and centrifuged at $13,000 \times g$ for $15 \mathrm{~min}$. The incubation with buffer 2 was repeated, until the pellet was completely translucent, at which point it was resuspended in $50 \mu \mathrm{l}$ of MQ. Purified S-layer samples were denatured and run on a precast gel (NuPAGE 4-12\% Bis-Tris Protein Gels). The protein band observed at $\sim 40 \mathrm{kDa}$ was excised (UniProt Acession: A0A0E3K5T7-slaB $=41 \mathrm{kDa}$ ), processed for mass spectrometric analysis and subsequent protein identification as described in ref. ${ }^{72}$.

Denaturing and reassembly of SlaA. S-layer was purified as described above and washed another five times in buffer 2, in order to remove SlaB from the S-layer preparation $^{38}$. The resultant S-layer extract was pelleted by centrifugation $(13,000 \times \mathrm{g}$ for $15 \mathrm{~min})$, resuspended in $20 \mathrm{mM} \mathrm{Na} \mathrm{CO}_{3}(\mathrm{pH} 10.5)$ as described in ref. ${ }^{45}$. The resuspended S-layer was incubated under agitation at room temperature overnight, after which the S-layer sacculi were no longer visible using Light Microscopy. In total, $10 \times$ concentrated Brock medium was added to the denatured 
proteins to a final concentration of $1 \times$, after which the $\mathrm{pH}$ was titrated to $\mathrm{pH} 3.5$ by the addition of $\mathrm{H}_{2} \mathrm{SO}_{4}$. The resulting solution was incubated overnight at $78^{\circ} \mathrm{C}$. After incubation, aggregates were observable.

Infection assay. Virion solutions for infection assays were prepared by sterile filtering (filter: $200 \mathrm{~nm}$ pore size) of supernatants of a SSV1-producing S. solfa taricus culture. Virion solutions were further verified to be pure of cell contaminants by microscopy (see above). Virus copy numbers in the virion solution were quantified by RT-qPCR using qD291FW/RV primers (see Quantitative RTPCR analysis and Supplementary Table 1). Fresh SB3 $\times 6$ and Ctrl-plasmid transformants were harvested at $\mathrm{OD}_{600}=0.1$ and adjusted to same cell numbers of $\sim 5 \times$ $10^{7}$ cells per $\mathrm{ml}$ (cell numbers assessed by FC, see Flow Cytometry) by transferring aliquots to pre-warmed medium, creating three replicate cultures. Each replicate was independently mixed with SSV1 virions at a distinct MOI $(0.1,5$, or $>100)$. As control, the same amount of the virus was added to cell-free medium and treated under same conditions. All cultures were incubated under standard conditions (78 ${ }^{\circ} \mathrm{C}$, shaking) and $1 \mathrm{ml}$ samples were taken at regular intervals, without interrupting the incubation process. Each sample was centrifuged for $10 \mathrm{~min}$ at $11,000 \times \mathrm{g}$ followed by separation of the supernatant from the cell pellet and further filtering of the supernatant ( $450 \mathrm{~nm}$ pore size). Cell pellets were washed with fresh Brock NZ/S medium (see Culturing) three times (centrifugation at $11,000 \times g$ ) to remove residual virus in the supernatant and finally resuspended in Brock NZ/S medium. In total, $5 \mu \mathrm{l}$ of filtered supernatant and the cell pellet solution respectively, were directly used as templates in separate RT-qPCR measurements (three technical replicates performed per biological replicate) each using qD291FW/RV primers (see Quantitative RT-PCR analysis and Supplementary table 1). Virus copy numbers measured in supernatants and resuspended cell pellets were normalized to those of initial virus added (as measured in the cell-free control) and to the cell count, which was assessed by FC (see Flow Cytometry) for each individual sample. For analysis, change of virion number was determined in comparison with timepoint $10 \mathrm{~min} \mathrm{PI}$.

Virus assays. For SSV1 infection assays at MOI 5, plaque assays were performed to assess the number of extracellular virions in supernatants sampled at different timepoints from infected SB3 $\times 6 /$ Ctrl plasmid transformants and cell-free controls (see Infection assay), respectively. Different dilutions of every supernatant sample were each mixed with $10 \times S$. solfataricus $\mathrm{P} 1$ in a $0.4 \%$ gellan gum solution and poured on Brock-medium plates (see Culturing). Plates were incubated for $48 \mathrm{~h}$ at $78{ }^{\circ} \mathrm{C}$. The number of plaques obtained from SB3 $\times 6$ and Ctrl supernatants was compared with the number of plaques present in a cell-free virus control. Spotting assays were performed by using different dilutions of SSV1 virion solutions (purified as described in "infection assay") and spotting them on lawns of $10 \times$ concentrated cells of SB3 66 and Ctrl plasmid cultures. Biological replicates represent independently incubated SB3 $\times 6$ and Ctrl cultures.

Reporting summary. Further information on research design is available in the Nature Research Reporting Summary linked to this article.

\section{Data availability}

The source data underlying Figs. 1b-f, 2c-e, 4, 5b-d, 6a, c and 7b, c, Table 1, and Supplementary Figs. $3 a, b, 4,7,11$, and $12 \mathrm{a}, \mathrm{b}$ are provided as a Source Data file and are available at figshare ${ }^{70}$.

Received: 20 February 2019; Accepted: 18 September 2019; Published online: 22 October 2019

\section{References}

1. Sleytr, U. B. Self-assembly of the hexagonally and tetragonally arranged subunits of bacterial surface layers and their reattachment to cell walls. $J$ Ultrastruct. Res. 55, 360-377 (1976).

2. Rodrigues-Oliveira, T., Belmok, A., Vasconcellos, D., Schuster, B. \& Kyaw, C. M. Archaeal S-layers: overview and current state of the art. Front. Microbiol. 8, 1-17 (2017).

3. Sleytr, U. B., Schuster, B., Egelseer, E. M. \& Pum, D. S-layers: principles and applications. FEMS Microbiol. Rev. 38, 823-864 (2014)

4. Fagan, R. P. \& Fairweather, N. F. Biogenesis and functions of bacterial Slayers. Nat. Rev. Microbiol. 12, 211-222 (2014).

5. Pohlschroder, M., Pfeiffer, F., Schulze, S. \& Halim, M. F. A. Archaeal cell surface biogenesis. FEMS Microbiol. Rev. 42, 694-717 (2018).

6. Baumeister, W. \& Lembcke, G. Structural features of archaebacterial cell envelopes. J. Bioenerg. Biomembr. 24, 567-575 (1992).

7. Li, P. N. et al. Nutrient transport suggests an evolutionary basis for charged archaeal surface layer proteins. ISME J. 12, 2389-2402 (2018).
8. Engelhardt, H. Are S-layers exoskeletons? The basic function of protein surface layers revisited. J. Struct. Biol. 160, 115-124 (2007).

9. Jarrell, K. F. et al. N-linked glycosylation in archaea: a structural, functional, and genetic anaysis. Microbiol. Mol. Biol. Rev. 78, 304-341 (2014).

10. Meyer, B. H. et al. Sulfoquinovose synthase - an important enzyme in the Nglycosylation pathway of Sulfolobus acidocaldarius. Mol. Microbiol. 82, 1150-1163 (2011)

11. Meyer, B. H. et al. Agl16, a thermophilic glycosyltransferase mediating the last step of N-Glycan biosynthesis in the Thermoacidophilic Crenarchaeon Sulfolobus acidocaldarius. J. Bacteriol. 195, 2177-2186 (2013).

12. Abu-Qarn, M. et al. Haloferax volcanii AglB and AglD are Involved in Nglycosylation of the S-layer glycoprotein and proper assembly of the surface layer. J. Mol. Biol. 374, 1224-1236 (2007).

13. Tamir, A. \& Eichler, J. N-Glycosylation is important for proper Haloferax volcanii S-layer stability and function. Appl. Environ. Microbiol. 83, e03152-16 (2017).

14. Wildhaber, I. \& Baumeister, W. The cell envelope of Thermoproteus tenax: three-dimensional structure of the surface layer and its role in shape maintenance. EMBO J. 6, 1475-1480 (1987).

15. Messner, P., Pum, D., Sára, M., Stetter, K. O. \& Sleytr, U. B. Ultrastructure of the cell envelope of the archaebacteria Thermoproteus tenax and Thermoproteus neutrophilus. J. Bacteriol. 166, 1046-1054 (1986).

16. Pum, D., Messner, P. \& Sleytr, U. B. Role of the S layer in morphogenesis and cell division of the archaebacterium Methanocorpusculum sinense. $J$ Bacteriol. 173, 6865-6873 (1991).

17. Mohr, V. \& Larsen, H. On the structural transformation and lysis of halobacterium salinarium in hypotonic and isotonic solutions. J. Gen. Microbiol 31, 267-280 (1996).

18. Jarrell, K. F., Colvin, J. R. \& Sprott, G. D. Spontaneous protoplast formation in Methanobacterium bryantii. J. Bacteriol. 149, 346-353 (1982).

19. Maslov, I. et al. Efficient non-cytotoxic fluorescent staining of halophiles. Sci. Rep. 8, 1-12 (2018).

20. Horikoshi, K., Aono, R. \& Nakamura, S. The triangular halophilic archaebacterium Haloarcula japonica strain TR-1. Experientia 49, 497-502 (1993).

21. Abdul Halim, M. F. et al. Haloferax volcanii archaeosortase is required for motility, mating, and C-terminal processing of the S-layer glycoprotein. Mol. Microbiol. 88, 1164-1175 (2013).

22. Abdul Halim, M. F. et al. Permuting the PGF signature motif blocks both archaeosortase-dependent c-terminal cleavage and prenyl lipid attachment for the haloferax volcanii s-layer glycoprotein. J. Bacteriol. 198, 808-815 (2015).

23. Zhang, C. et al. Cell structure changes in the hyperthermophilic crenarchaeon Sulfolobus islandicus lacking the S-layer. mBio 10, e01589-19 (2019).

24. Zhang, C., Phillips, A. P. R., Wipfler, R. L., Olsen, G. J. \& Whitaker, R. J. The essential genome of the crenarchaeal model Sulfolobus islandicus. Nat. Commun. 9, 4908 (2018).

25. Engelhardt, H. Mechanism of osmoprotection by archaeal S-layers: a theoretical study. J. Struct. Biol. 160, 190-199 (2007).

26. Leigh, J. A., Albers, S. V., Atomi, H. \& Allers, T. Model organisms for genetics in the domain archaea: methanogens, halophiles, thermococcales and sulfolobales. FEMS Microbiol. Rev. 35, 577-608 (2011).

27. McDougall, M. et al. Archaea S-layer nanotube from a "black smoker" in complex with cyclo-octasulfur (S 8) rings. Proteins Struct. Funct. Bioinformatics. 85, 2209-2216 (2017).

28. Kish, A. et al. Preservation of archaeal surface layer structure during mineralization. Sci. Rep. 6, 26152 (2016)

29. Shalev, Y., Turgeman-Grott, I., Tamir, A., Eichler, J. \& Gophna, U. Cell surface glycosylation is required for efficient mating of Haloferax volcanii. Front. Microbiol. 8, 1-8 (2017).

30. Shalev, Y. et al. Comparative analysis of surface layer glycoproteins and genes involved in protein glycosylation in the genus haloferax. Genes (Basel). 9, pii: E172 (2018)

31. Zaretsky, M., Roine, E. \& Eichler, J. Sialic acid-like sugars in archaea: legionaminic acid biosynthesis in the Halophile Halorubrum sp. PV6. Front. Microbiol. 9, 2133 (2018).

32. Kandiba, L. et al. Diversity in prokaryotic glycosylation: an archaeal-derived Nlinked glycan contains legionaminic acid. Mol. Microbiol. 84, 578-593 (2012).

33. Quemin, E. R. J. et al. Eukaryotic-like virus budding in archaea. MBio 7, 3-7 (2016).

34. Zebec, Z., Manica, a., Zhang, J., White, M. F. \& Schleper, C. CRISPR-mediated targeted mRNA degradation in the archaeon Sulfolobus solfataricus. Nucleic Acids Res. 42, 5280-5288 (2014)

35. Zebec, Z., Zink, I. A., Kerou, M. \& Schleper, C. Efficient CRISPR-mediated post-transcriptional gene silencing in a hyperthermophilic archaeon using multiplexed crRNA expression. G3 6, 3161-3168 (2016)

36. Peng, W., Feng, M., Feng, X., Liang, Y. X. \& She, Q. An archaeal CRISPR type III-B system exhibiting distinctive RNA targeting features and mediating dual RNA and DNA interference. Nucleic Acids Res. 43, 406-417 (2014). 
37. Han, W., Feng, X. \& She, Q. Reverse gyrase functions in genome integrity maintenance by protecting DNA breaks in vivo. 18, 1340 (2017).

38. Veith, A. et al. Acidianus, Sulfolobus and Metallosphaera surface layers: structure, composition and gene expression. Mol. Microbiol. 73, 58-72 (2009).

39. Palmieri, G. et al. Surface-exposed glycoproteins of Hyperthermophilic Sulfolobus solfataricus $\mathrm{P} 2$ show a common $\mathrm{N}$-glycosylation Profile. J. Proteome Res. 12, 2779-2790 (2013).

40. Banerjee, A. et al. FlaF is a $\beta$-sandwich protein that anchors the archaellum in the archaeal cell envelope by binding the S-layer protein. Structure 23, 863-872 (2015).

41. Zolghadr, B., Klingl, A., Rachel, R., Driessen, A. J. M. \& Albers, S.-V. The bindosome is a structural component of the Sulfolobus solfataricus cell envelope. Extremophiles 15, 235-244 (2011).

42. Berkner, S., Wlodkowski, A., Albers, S.-V. \& Lipps, G. Inducible and constitutive promoters for genetic systems in Sulfolobus acidocaldarius. Extremophiles 14, 249-259 (2010).

43. Zink, I. A. Development of a plasmid shuttle vector system for CRISPR studies in the archaeon Sulfolobus solfataricus. (University of Vienna, Vienna, 2013).

44. Liu, J. et al. Functional assignment of multiple ESCRT-III homologs in cell division and budding in Sulfolobus islandicus. Mol. Microbiol. 105, 540-553 (2017).

45. Weiss, R. L. Subunit cell wall of Sulfolobus acidocaldarius. J. Bacteriol. 118, 275-284 (1974).

46. Meyer, B. H. \& Albers, S.-V. Hot and sweet: protein glycosylation in Crenarchaeota. Biochem. Soc. Trans. 41, 384-392 (2013).

47. Firtel, M., Patel, G. B. \& Beveridge, T. J. S layer regeneration in Methanococcus voltae protoplasts. Microbiology 141, 817-824 (1995).

48. Hjort, K. \& Bernander, R. Cell cycle regulation in the hyperthermophilic crenarchaeon Sulfolobus acidocaldarius. Mol. Microbiol. 40, 225-234 (2001).

49. Ceballos, R. M. et al. Differential virus host-ranges of the Fuselloviridae of hyperthermophilic Archaea: implications for evolution in extreme environments. Front. Microbiol. 3, 1-10 (2012).

50. Osawa, T., Inanaga, H., Sato, C. \& Numata, T. Crystal Structure of the CRISPR-Cas RNA Silencing Cmr Complex Bound to a Target Analog. Mol. Cell 58, 1-14 (2015).

51. Manica, A., Zebec, Z., Steinkellner, J. \& Schleper, C. Unexpectedly broad target recognition of the CRISPR-mediated virus defence system in the archaeon Sulfolobus solfataricus. Nucleic Acids Res. 41, 1, -9 (2013).

52. Rouillon, C., Athukoralage, J. S., Graham, S., Grüschow, S. \& White, M. F. Control of cyclic oligoadenylate synthesis in a type III CRISPR system. Elife 7, pii: e36734 (2018).

53. Fellmann, C. \& Lowe, S. W. Stable RNA interference rules for silencing. Nat. Cell Biol. 16, 10-18 (2014).

54. Ellen, A. F., Albers, S. V. \& Driessen, A. J. M. Comparative study of the extracellular proteome of Sulfolobus species reveals limited secretion. Extremophiles 14, 87-98 (2009).

55. Gorlas, A. et al. Sulfur vesicles from Thermococcales: A possible role in sulfur detoxifying mechanisms. Biochimie 118, 356-364 (2015).

56. Guan, Z. et al. N-glycosylation in the thermoacidophilic archaeon Sulfolobus acidocaldarius involves a short dolichol pyrophosphate carrier. FEBS Lett. 590, 3168-3178 (2016).

57. Schuster, B. \& Sleytr, U. B. Relevance of glycosylation of S-layer proteins for cell surface properties. Acta Biomater. 19, 149 (2015).

58. Jarrell, K. F., Jones, G. M. \& Nair, D. B. Biosynthesis and role of N-linked glycosylation in cell surface structures of archaea with a focus on flagella and $s$ layers. Int. J. Microbiol. 2010, 470138 (2010).

59. Kiener, A., Konig, H., Winter, J. \& Leisinger, T. Purification and use of Methanobacterium wolfei pseudomurein endopeptidase for lysis of Methanobacterium thermoautotrophicum. J. Bacteriol. 169, 1010-1016 (1987).

60. Samson, R. Y., Obita, T., Freund, S. M., Williams, R. L. \& Bell, S. D. A role for the ESCRT system in cell division in archaea. Science 322, 1710-1713 (2008).

61. Yang, N. \& Driessen, A. J. M. Deletion of cdvB paralogous genes of Sulfolobus acidocaldarius impairs cell division. Extremophiles 18, 331-339 (2014).

62. Long, S. W. \& Faguy, D. M. Anucleate and titan cell phenotypes caused by insertional inactivation of the structural maintenance of chromosomes (smc) gene in the archaeon Methanococcus voltae. Mol. Microbiol. 52, 1567-1577 (2004).

63. Dobro, M. J. et al. Electron cryotomography of ESCRT assemblies and dividing Sulfolobus cells suggests that spiraling filaments are involved in membrane scission. Mol. Biol. Cell 24, 2319-2327 (2013).

64. Prangishvili, D. et al. The enigmatic archaeal virosphere. Nat. Rev. Microbiol. 15, 724-739 (2017).
65. Uldahl, K. B. et al. Life cycle characterization of sulfolobus monocaudavirus 1 , an extremophilic spindle-shaped virus with extracellular tail development. J. Virol. 90, 5693-5699 (2016).

66. Martin, A. et al. SAV 1, a temperate u.v.-inducible DNA virus-like particle from the archaebacterium Sulfolobus acidocaldarius isolate B12. EMBO J. 3 , 2165-2168 (1984).

67. Bertozzi Silva, J., Storms, Z. \& Sauvageau, D. Host receptors for bacteriophage adsorption. FEMS Microbiol. Lett. 363, 1-11 (2016).

68. Fröls, S. et al. UV-inducible cellular aggregation of the hyperthermophilic archaeon Sulfolobus solfataricus is mediated by pili formation. Mol. Microbiol. 70, 938-952 (2008).

69. van Wolferen, M., Orell, A. \& Albers, S.-V. Archaeal biofilm formation. Nat Rev. Microbiol. 16, 699-713 (2018).

70. Zink, I. A., Pfeifer, K. \& Schleper, C. ZinkPfeifer_Source_Data.xlsx. (2019). https://doi.org/10.6084/m9.figshare.9772511.v1

71. Ducret, A., Quardokus, E. M. \& Brun, Y. V. MicrobeJ, a tool for high throughput bacterial cell detection and quantitative analysis. Nat. Microbiol. 1, 16077 (2016).

72. Ott, E. et al. Proteometabolomic response of Deinococcus radiodurans exposed to UVC and vacuum conditions: Initial studies prior to the Tanpopo space mission. PLoS ONE 12, e0189381 (2017).

\section{Acknowledgements}

We thank Stephan Köstlbacher for help with data imaging, as well as Melina Kerou and Filipa Sousa for technical advice and scientific discussions. EM work was performed at the Core Facility Cell Imaging and Ultrastructure Research, University of Vienna-member of the Vienna Life-Science Instruments (VLSI) with special thanks to Norbert Cyran, Daniela Gruber and Siegfried Reipert. Also, special thanks go to all members of the master course "MMEI-(300 307)" 2017, who provided first results on the silencing experiment. This work was financed through a DOC-fellowship of the Austrian Academy of Sciences (OEAW) and a PhD completion grant of the University of Vienna to IZ, through P 29399-B22 of the Austrian Science Fund (FWF) to BS, as well as ERCAdv grant TACKLE (No. 695192) to CS.

\section{Author contributions}

I.Z., K.P., and E.W. performed experiments, C.S. and I.Z. conceived this study, I.Z. and K.P. wrote first manuscript draft, C.S., I.Z., K.P., B.S., E.W., and U.S. discussed results and manuscript.

\section{Competing interests}

The authors declare no competing interests.

\section{Additional information}

Supplementary information is available for this paper at https://doi.org/10.1038/s41467 019-12745-x.

Correspondence and requests for materials should be addressed to C.S.

Peer review information Nature Communications thanks the anonymous reviewers for their contribution to the peer review of this work. Peer reviewer reports are available.

Reprints and permission information is available at http://www.nature.com/reprints

Publisher's note Springer Nature remains neutral with regard to jurisdictional claims in published maps and institutional affiliations.

Open Access This article is licensed under a Creative Commons Attribution 4.0 International License, which permits use, sharing, adaptation, distribution and reproduction in any medium or format, as long as you give appropriate credit to the original author(s) and the source, provide a link to the Creative Commons license, and indicate if changes were made. The images or other third party material in this article are included in the article's Creative Commons license, unless indicated otherwise in a credit line to the material. If material is not included in the article's Creative Commons license and your intended use is not permitted by statutory regulation or exceeds the permitted use, you will need to obtain permission directly from the copyright holder. To view a copy of this license, visit http://creativecommons.org/ licenses/by/4.0/

(C) The Author(s) 2019 